\title{
Conformal bridge in a cosmic string background
}

\author{
Luis Inzunza and Mikhail S. Plyushchay \\ Departamento de Física, Universidad de Santiago de Chile, \\ Casilla 30\%, Santiago, Chile \\ E-mail: luis.inzunza@usach.cl, mikhail.plyushchay@usach.cl
}

ABSTRACT: Hidden symmetries of non-relativistic $\mathfrak{s o}(2,1) \cong \mathfrak{s l}(2, \mathbb{R})$ invariant systems in a cosmic string background are studied using the conformal bridge transformation. Geometric properties of this background are analogous to those of a conical surface with a deficiency/excess angle encoded in the "geometrical parameter" $\alpha$, determined by the linear positive/negative mass density of the string. The free particle and the harmonic oscillator on this background are shown to be related by the conformal bridge transformation. To identify the integrals of the free system, we employ a local canonical transformation that relates the model with its planar version. The conformal bridge transformation is then used to map the obtained integrals to those of the harmonic oscillator on the cone. Well-defined classical integrals in both models exist only at $\alpha=q / k$ with $q, k=1,2, \ldots$, which for $q>1$ are higher-order generators of finite nonlinear algebras. The systems are quantized for arbitrary values of $\alpha$; however, the well-defined hidden symmetry operators associated with spectral degeneracies only exist when $\alpha$ is an integer, that reveals a quantum anomaly.

KEYwORDS: Conformal and W Symmetry, String theory and cosmic strings

ArXiv EPrint: 2012.04613 


\section{Contents}

1 Introduction 1

2 Cosmic string and conical geometry 3

3 Dynamics in the Euclidean plane $\quad 7$

$\begin{array}{ll}3.1 \text { The free particle } & 7\end{array}$

$\begin{array}{ll}3.2 & \text { The isotropic harmonic oscillator } \\ \end{array}$

4 The conformal bridge transformation $\quad 15$

5 Free motion in a cosmic string background $\quad 19$

$\begin{array}{ll}5.1 \text { Classical case } & 19\end{array}$

5.2 Quantum case 24

6 Harmonic oscillator in a cosmic string background 27

$\begin{array}{lll}6.1 \text { Classical case } & 27\end{array}$

$\begin{array}{ll}6.2 \text { Quantum case } & 30\end{array}$

$\begin{array}{lll}7 & \text { Discussion and outlook } & 35\end{array}$

\section{Introduction}

Special characteristics of classical systems are reflected by the conserved quantities that canonically generate the symmetry transformations. At the quantum level, these quantities are promoted to the operators that carry the spectrum information. In this context, hidden symmetries are associated with the non-obvious integrals of motion that mix canonical coordinates and momenta in a non-trivial way at the classical level, while at the quantum level they are often responsible for the so-called "accidental degenerations" [1]. Integrals of this type are higher-order functions of the canonical momenta, and they may generate nonlinear symmetry algebras $[2,3]$. Some examples of integrals related to hidden symmetries are the Laplace-Runge-Lenz vector for the Kepler-Coulomb problem [4], the Fradkin tensor for the isotropic harmonic oscillator [5], the analogs of these integrals in a monopole background $[6,7]$, the higher-order symmetry generators of the anisotropic harmonic oscillator with commensurable frequencies [2], and the $N$ integrals in involution in the Calogero models of $N$ particles $[8,9]$.

On the other hand, it is a quite general assertion that the systems in a curved spacetime show special properties related to the geometric background itself. Some examples in this direction are the Hawking radiation [10], the Unruh effect [11], the conformal invariance of a charged particle propagating near the horizon of the extreme Reissner-Nordström black 
hole, which attracted attention in the context of AdS/CFT correspondence [12-15] (that, in turn, gave rise to a resurgence of interest to the conformal model of de Alfaro, Fubini and Furlan [16]). In a different but related line of research we also mention here the supersymmetric mechanics models on curved spaces whose construction is based on the WDVV equation formalism $[17,18]$.

In the context of the present research, the study of classical and quantum dynamics in curved spaces is interesting from the point of view of hidden symmetries. An important result in this direction is the proof of the existence of a non-trivial conserved quantity that characterizes the dynamics of a particle moving on a Kerr black hole background. This quantity, known as the Carter integral [19], is responsible for the complete integrability of the system. Another interesting result was reported in [20], where it was shown that spinning particles in the Kerr-Newman black hole background are characterized by enhanced supersymmetry with additional supercharges of a nature different from a square root of the Hamiltonian of the system. Geometrically, such supercharges are associated with the so-called Yano and conformal Yano tensors [21-24]. Some other examples related to the particle dynamics in curved spaces can be found in references [25-35].

In this article, we address the problem of studying the classical and quantum symmetry aspects of some conformal-invariant non-relativistic particle systems on a cosmic string background. These strings are topological defects whose creation in the early universe is predicted by quantum field theory arguments [36-39], and which, on the other hand, also appear in condensed matter physics and wormholes [40-44]. Their effect is to introduce a conical singularity in the spatial part of the space-time metric [43, 45].

The effects of the presence of the cosmic string was examined in different physical contexts, see, e.g., refs. [27, 31, 33-35, 46-50]. Our goal here is to study the influence of the geometrical properties of this background (encoded in a "geometrical parameter" $\alpha$ given in terms of the linear mass density of the string) on the dynamics of the systems from the perspective of well-defined integrals of motion in the phase space when considering classical cases, and well-defined symmetry operators for the corresponding quantum versions of the systems. We are interested in the case of the free particle in the cone, which has the $\mathfrak{s o}(2,1)$ conformal symmetry, and in the harmonic oscillators system in the same geometry, which is characterized by the $\mathfrak{s l}(2, \mathbb{R}) \cong \mathfrak{s o}(2,1)$ conformal Newton-Hooke symmetry [51-55]. The key construction in our investigation is the so-called conformal bridge transformation [55], which in general is a mapping that allows us to transform the complete set of symmetry generators from an $\mathfrak{s o}(2,1)$ invariant asymptotically free system, to those of a harmonically confined model with the $\mathfrak{s l}(2, \mathbb{R})$ conformal symmetry. The plan is to first characterize the free case by identifying the corresponding classical and quantum integrals of motion for different values of $\alpha$, and then to obtain the complete information on the harmonic oscillator system through the conformal bridge transformation.

The free dynamics and the harmonic oscillator system in conical geometry were already studied in the literature, but under a different perspective. In $[25,26]$ the classical and quantum scattering on the cone were considered. There, in particular, it was noticed that for the geodesic motion, there are special values of the deficiency angle for which the particle experiences a backward scattering, while for other values the particle continues to move in 
the original direction, making several revolutions around the cone vertex. For the harmonic oscillator system, the structure of the wave-functions and the spectrum for arbitrary values of $\alpha$ were investigated in [28, 29, 32]. None of these works, however, studied the systems in the light of hidden symmetries which appear for special values of $\alpha$.

A notable result we present here is that for the free particle on the cone characterized by the parameter $\alpha$, there are well-defined integrals of motion in the phase space only when $\alpha$ is a rational number. In the general case of rational $\alpha$ they are of higher-order in momenta and produce a finite nonlinear algebra. At the quantum level we reveal a quantum anomaly, because although the system can be quantized for any real value of $\alpha$, the welldefined hidden symmetry operators in Hilbert physical space can only be constructed when this geometric parameter is an integer. The same peculiarities appear for the harmonic oscillator system, whose classical trajectories are closed for rational values of $\alpha$, and at the quantum level, only the cases with its integer values are anomaly-free, and the hidden symmetry operators reflect then the corresponding degeneracies of the spectrum.

The article is organized as follows. We first review the geometry related to a cosmic string background in section 2 by explicitly showing that its spatial part takes the form of a two-dimensional conical metric, whose parameter is defined by the mass density of the string. We also show that there is a set of local coordinates which allows us to formally relate the conical metric to that of the Euclidean plane. In section 3, we consider some important aspects of the non-relativistic free particle and the harmonic oscillator dynamics in $\mathbb{R}^{2}$. This section serves as the basis from which we can build the symmetry algebra of the corresponding versions of these systems in conical geometry by using a locally defined canonical transformation. In section 4 , we explain how the conformal bridge transformation works. As an example, it is shown how to relate the Euclidean free particle to the planar isotropic harmonic oscillator by means of this mapping. We also establish there the nontrivial relation between the hidden symmetry generators of these two systems and some sub-algebras generated by them. In section 5 we study the free particle on a cosmic string background. Using the above-mentioned canonical transformation, we obtain the explicit form of the solutions of the equations of motion, as well as some conserved quantities which are generally of a formal nature, but which serve to construct well-defined integrals in the phase space when the geometric parameter is a rational number. At the quantum level, we construct the symmetry operators and observe the quantum anomaly by analyzing the action of these operators on the physical eigenstates. In section 6 , we employ the conformal bridge transformation to get the symmetry generators of the harmonic oscillator from the free particle system in the classical and quantum cases. The properties of symmetry algebra for the harmonically confined system, as well as the manifestation of the same quantum anomaly are immediately obtained by using the same transformation. Finally, in section 7 the discussion of the obtained results and outlook are presented.

\section{Cosmic string and conical geometry}

Cosmic strings are hypothetical one-dimensional topological defects which may have formed in the spontaneous symmetry-breaking phase transition in the expanding Universe [36-39]. 
Such defects also are familiar in condensed matter physics $[42,43]$. In this article we are interested in analyzing the symmetries of a non-relativistic conformal dynamics, at the classical and quantum levels, of a particle in a two-dimensional space with properties of a cosmic string background. In this section, we show that this problem is analogous to studying the dynamics of a particle in a conical geometry [25, 26], and discuss some of its general properties which will be important for the subsequent analysis.

Following [38, 45], the solution of the Einstein field equations in $(2+1)$ dimensions associated with cosmic string is described by the metric

$$
\begin{aligned}
d S^{2} & =-c^{2} d t^{2}+d s^{2}, \\
d s^{2} & =\left(1-\frac{8 \mu G}{c^{2}} \ln \left(\frac{r}{r_{0}}\right)\right)\left(d r^{2}+r^{2} d \varphi^{2}\right),
\end{aligned}
$$

where $G$ is the Newton constant, $c$ is the speed of light, $\mu$ is the linear mass density of the cosmic string, and $r_{0}$ corresponds to its radius. By introducing the new coordinate

$$
\begin{aligned}
r^{2} & =\left(1-\frac{8 \mu G}{c^{2}} \ln \left(\frac{r}{r_{0}}\right)\right) r^{2}, \quad \alpha^{2} d r^{2}=\left(1-\frac{8 \mu G}{c^{2}} \ln \left(\frac{r}{r_{0}}\right)\right) d r^{2}, \\
\alpha & =\frac{1}{1-\frac{4 \mu G}{c^{2}}}>0,
\end{aligned}
$$

where higher-order terms in $\mu G / c^{2}$ are neglected in the computation of $d r^{\prime 2}$, the spatial part (2.2) of the cosmic string metric (2.1) takes the form

$$
d s^{2}=\alpha^{2} d r^{2}+r^{2} d \varphi^{2}
$$

after changing the notation $r^{\prime} \rightarrow r$. Eq. (2.5) corresponds to the two-dimensional metric of a conical geometry. For $\alpha>1$, it can be obtained by reducing the three-dimensional Euclidean metric

$$
d s_{E}^{2}=d r^{2}+r^{2} d \varphi^{2}+d z^{2},
$$

to the surface of a cone $z=\lambda r$, and identification $\alpha^{2}=1+\lambda^{2}$. Here $\lambda=\cot \beta, \beta$ is the aperture angle of the cone, and in accordance with (2.4), this metric is associated to a cosmic string whose mass density is positive. On the other hand, the metric (2.5) with $0<\alpha<1$ can be obtained from the $(2+1)$-dimensional Minkowski space

$$
d s_{M}^{2}=-c^{2} d \tau^{2}+d r^{2}+r^{2} d \varphi^{2}
$$

by reduction to the cone surface $c \tau=\lambda r, 0<\lambda<1, \alpha^{2}=1-\lambda^{2}$. Formally, this is the reduction to the non-causal part of Minkowski space, where particle's velocity is greater than $c$. In correspondence with (2.4), these spaces are associated with cosmic strings that have negative mass density [40]. The case of the conic metric with $0<\alpha<1$ also describes topological defects in the physics of condensed matter and wormholes [40-44].

From here one sees that studying a free non-relativistic particle system in a cosmic string background geometry with a given value of the parameter $\alpha$ is analogous to solving the problem of the dynamics of a particle on the surface of a cone described by the action 
$S=-m c^{2} \int \sqrt{1-\frac{1}{c^{2}}\left(\frac{d s}{d t}\right)^{2}} d t$ in the non-relativistic limit $c \rightarrow \infty$. In the remainder of the section, we explore some geometric properties of the conical space (2.5). For this, we express the metric $d s^{2}$ in different coordinate systems, that will allow us to clearly see the singularity at the origin, and clarify the corresponding conformal properties of the metric (2.1).

First, we note that in Cartesian coordinates,

$$
d s^{2}=\frac{1}{\left(x^{2}+y^{2}\right)}\left(\alpha^{2}(x d x+y d y)^{2}+(x d y-y d x)^{2}\right),
$$

the singularity of the metric at the origin $x=y=0$ for $\alpha \neq 1$ becomes apparent.

Introducing a new radial coordinate $r=r_{0} e^{\frac{\rho}{\alpha}}$, the metric becomes

$$
d s^{2}=r_{0}^{2} e^{\frac{2 \rho}{\alpha}}\left(d \rho^{2}+d \varphi^{2}\right) .
$$

The variables $\rho$ and $\varphi$ correspond to the isothermal coordinates, and we note that when $\alpha \rightarrow \infty$, the metric (2.9) transforms into a cylinder's metric. From (2.9), as well as from (2.5), the invariance of the metric under rotations, $\varphi \rightarrow \varphi+\varphi_{0}$, is obvious. The corresponding Killing vector is $\frac{\partial}{\partial \varphi}$. Metric (2.9) also is conformally invariant under transformations $\rho \rightarrow \rho+\rho_{0}$. In polar coordinates, this corresponds to dilatation in the radial coordinate generated by the conformal Killing vector $\frac{\partial}{\partial \rho}=\frac{r}{\alpha} \frac{\partial}{\partial r}$.

By introducing a pair of "regularized" Cartesian coordinates

$$
X_{1}=\alpha r \cos \Phi, \quad X_{2}=\alpha r \sin \Phi, \quad \Phi=\varphi / \alpha,
$$

the metric (2.5) is transformed into

$$
d s^{2}=d X_{1}^{2}+d X_{2}^{2} .
$$

This formally looks like the metric of the Euclidean plane in Cartesian coordinates, but $0 \leq \Phi<2 \pi / \alpha$ in (2.10), and the edges $\Phi=0$ and $\Phi=2 \pi / \alpha$ have to be identified, that results in a conical singularity. This singularity reveals itself, particularly, in Riemann curvature tensor concentrated at $r=0: \mathcal{R}_{r \varphi}^{r \varphi}=2 \pi\left(1-\alpha^{-1}\right) \delta\left(X_{1}\right) \delta\left(X_{2}\right)[43,45]$.

To clarify further the nature of coordinates $(2.10)$, consider the complex combination

$$
w=X_{1}+i X_{2}=\alpha r e^{i \varphi / \alpha}, \quad d s^{2}=d w d w^{*} .
$$

From here it is seen that there may be problems for arbitrary values of $\alpha$ due to the exponential factor and the associated branch point. When the rational case $\alpha=q / k$ with $q, k=1,2, \ldots$, is considered, one can use instead the new coordinates $\zeta=\zeta_{1}+i \zeta_{2}$,

$$
\zeta=w^{q}=\left(\frac{q r}{k}\right)^{q} e^{i k \varphi} \Rightarrow \zeta_{1}=\left(\frac{q r}{k}\right)^{q} \cos (k \varphi), \quad \zeta_{2}=\left(\frac{q r}{k}\right)^{q} \sin (k \varphi) .
$$

In their terms the metric reads

$$
d s^{2}=\frac{d \zeta_{1}^{2}+d \zeta_{2}^{2}}{q^{2}\left(\zeta_{1}^{2}+\zeta_{2}^{2}\right)^{1-\frac{1}{q}}}
$$


For $q=1 \Rightarrow \alpha=1 / k$, it seems that there is no singularity in the metric, and the coordinates $\zeta_{1}, \zeta_{2}$ themselves reveal no singularity. However, as it is seen from (2.13), in this case $\zeta_{1}$ and $\zeta_{2}$ cover conical space $k$ times when $\alpha=1 / k$. The picture is similar to a Riemann surface for the function $w=z^{1 / k}$ where we pass from its one sheet to another when angle increases in $2 \pi$, while here the transition from one sheet to another happens each time when $\varphi$ increases in $2 \pi / k$.

Note also here that formally metric (2.11) is invariant under translations $X_{i} \rightarrow X_{i}+a_{i}$, $i=1,2$, produced by the vector fields

$$
\frac{\partial}{\partial X_{1}}=\frac{1}{\alpha} \cos \left(\frac{\varphi}{\alpha}\right) \frac{\partial}{\partial r}-\frac{1}{r} \sin \left(\frac{\varphi}{\alpha}\right) \frac{\partial}{\partial \varphi}, \quad \frac{\partial}{\partial X_{2}}=\frac{1}{\alpha} \sin \left(\frac{\varphi}{\alpha}\right) \frac{\partial}{\partial r}+\frac{1}{r} \cos \left(\frac{\varphi}{\alpha}\right) \frac{\partial}{\partial \varphi} .
$$

The infinitesimal $\left(\delta_{1}, \delta_{2} \sim 0\right)$ form of transformations generated by $(2.15)$ is

$$
\begin{array}{llll}
\frac{\partial}{\partial X_{1}}: & \Rightarrow & r \rightarrow r_{1}^{\prime}=r+\frac{\delta_{1}}{\alpha} \cos \left(\frac{\varphi}{\alpha}\right), & \varphi \rightarrow \varphi_{1}^{\prime}=\varphi-\frac{\delta_{1}}{r} \sin \left(\frac{\varphi}{\alpha}\right), \\
\frac{\partial}{\partial X_{2}}: & \Rightarrow & r \rightarrow r_{2}^{\prime}=r+\frac{\delta_{2}}{\alpha} \sin \left(\frac{\varphi}{\alpha}\right), & \varphi \rightarrow \varphi_{2}^{\prime}=\varphi+\frac{\delta_{2}}{r} \cos \left(\frac{\varphi}{\alpha}\right) .
\end{array}
$$

In spite of that these formal transformations are local isometries, one sees their singular nature at $r=0$ when $\alpha \neq 1 / k$. Furthermore, the corresponding global transformations,

$$
\begin{aligned}
& r^{2} \rightarrow\left(r_{1}^{\prime}\right)^{2}=r^{2}+\frac{2 \delta_{1} r}{\alpha} \cos \left(\frac{\varphi}{\alpha}\right)+\frac{\delta_{1}^{2}}{\alpha^{2}}, \quad \varphi \rightarrow \varphi_{1}^{\prime}=\alpha \arctan \left(\frac{\alpha r \sin \left(\frac{\varphi}{\alpha}\right)}{\alpha r \cos \left(\frac{\varphi}{\alpha}\right)+\delta_{1}}\right), \\
& r^{2} \rightarrow\left(r_{2}^{\prime}\right)^{2}=r^{2}+\frac{2 \delta_{2} r}{\alpha} \sin \left(\frac{\varphi}{\alpha}\right)+\frac{\delta_{2}^{2}}{\alpha^{2}}, \quad \varphi \rightarrow \varphi_{2}^{\prime}=\alpha \arctan \left(\frac{\alpha r \sin \left(\frac{\varphi}{\alpha}\right)+\delta_{2}}{\alpha r \cos \left(\frac{\varphi}{\alpha}\right)}\right),
\end{aligned}
$$

reveal their singularity for $\alpha \neq 1$.

The space-time metric (2.1) with spatial part presented in the form (2.11) looks like the metric of (2+1)-dimensional Minkowski space $d S^{2}=\eta_{\mu \nu} d X^{\mu} d X^{\nu}, X^{0}=c t$, $\eta_{\mu \nu}=\operatorname{diag}(-1,1,1)$. Locally, it is conformally invariant under transformations of the conformal $\mathrm{SO}(3,2)$ group, whose classical generators are $P^{\mu}, J^{\mu \nu}=X^{\mu} P^{\nu}-X^{\nu} P^{\mu}$, $K^{\mu}=2 X^{\mu}(X P)-X^{2} P^{\mu}$ and $D=X P$, where $P_{\mu}=\eta_{\mu \nu} P^{\nu}$ are the momenta canonically conjugate to $X^{\mu}$. Taking into account that $P_{1}=\frac{1}{\alpha} p_{r} \cos (\varphi / \alpha)-\alpha p_{\varphi} \sin (\varphi / \alpha)$, $P_{2}=\frac{1}{\alpha} p_{r} \sin (\varphi / \alpha)+\alpha p_{\varphi} \cos (\varphi / \alpha)$, where $p_{r}$ and $p_{\varphi}$ are the momenta canonically conjugate to $r$ and $\varphi$, one finds that only the generators of the time translation, $P^{0}$, the spatial rotation, $J^{12}=p_{\varphi}$, the dilatations, $D=-X^{0} P^{0}+r p_{r}$, and special conformal transformations, $K^{0}=2 X^{0} D-\left(\alpha^{2} r^{2}-\left(X^{0}\right)^{2}\right) P^{0}$, are globally well-defined for arbitrary values of the parameter $\alpha$, while generators of the spatial translations, $P^{i}$, Lorentz boosts, $J^{0 i}$, and generators of special conformal transformations, $K^{i}$, are globally well-defined only for $\alpha=1 / k$. After the appropriately taken non-relativistic limit [56-62], as we shall see, the rotation generator $p_{\varphi}$ and the corresponding analogs of the generators $P^{0}, D$ and $K^{0}$ will play the key role in our subsequent analysis. At the same time, in spite of the globally not well-defined nature (in the general case of the parameter $\alpha$ values) of generators of the spatial translations and Lorentz boosts, their corresponding non-relativistic analogs will be employed by us for the construction of the globally well-defined generators of the hidden symmetries. 
The change of coordinates (2.10) and the geodesic analysis presented in section 5 will show that the non-relativistic dynamics in the cosmic string background can be related to the free motion in the Euclidean plane. Bearing this in mind, instead of jumping directly to the analysis of the dynamics in the conical geometry, it is appropriate to review some important characteristics related to the motion in $\mathbb{R}^{2}$.

\section{Dynamics in the Euclidean plane}

To understand the complete symmetry algebra of a given mechanical system in a cosmic string (conical) background, both at the classical and quantum levels, it is instructive to remind the corresponding properties of such a system in the flat Euclidean plane. Later on, we will show that there is a formal canonical transformation related to the change of coordinates (2.10) which allows us to connect the dynamics in conical geometry with the corresponding dynamics in $\mathbb{R}^{2}$. We are interested in the free particle dynamics as well as the dynamics of the particle in the harmonic trap, so this section contains all we need to know of these two systems in the Euclidean plane.

\subsection{The free particle}

Here we present the complete set of integrals of motion of order not higher than two in momenta and display their explicit Lie algebra for a particle in Euclidean plane. Next we use the conserved quantities to reconstruct the trajectory of the particle. Finally we briefly describe the quantum theory of the system using the polar coordinates.

The quadratic in momenta and coordinates integrals of motion are

$$
\begin{aligned}
H & =\frac{1}{2 m} p_{+} p_{-}, & D & =\frac{1}{4}\left(\chi_{+} p_{-}+p_{+} \chi_{+}\right), \quad K=\frac{m}{2} \chi_{+} \chi_{-}, \\
J_{0} & =\frac{i}{4}\left(\chi_{+} p_{-}-p_{+} \chi_{-}\right), & J_{ \pm} & =\frac{1}{2} \chi_{ \pm} p_{ \pm}, \\
T_{ \pm} & =\frac{1}{2 m}\left(p_{ \pm}\right)^{2}, & S_{ \pm} & =\frac{m}{2}\left(\chi_{ \pm}\right)^{2}
\end{aligned}
$$

where

$$
p_{ \pm}=p_{1} \pm i p_{2}, \quad \chi_{ \pm}=\chi_{1} \pm i \chi_{2}
$$

are the complex combinations of the canonical momenta $p_{i}$ and the Galilean boost generators $\chi_{i}=x_{i}-\frac{1}{m} p_{i} t$.

The Hamiltonian $H$, the angular momentum $p_{\varphi}=2 J_{0}$, and the integrals $p_{ \pm}, T_{ \pm}$are the conserved quantities not depending explicitly on time $t$. The integrals $J_{0}, p_{ \pm}$and $\chi_{ \pm}$, unlike the rest of the listed integrals, do not mix coordinates $x_{i}$ and momenta $p_{i}$ when they act in the phase space via Poisson brackets. The integrals $H, D$ and $K$ correspond to the planar case $\alpha=1$ of the non-relativistic limit of the generators $P^{0}, D$ and $K^{0}$ mentioned in the previous section, while the Galilean boost generators $\chi_{i}$ appear as the non-relativistic limit of the Lorentz boosts $J^{0 i}$, see refs. [57, 58,61]. Note that the integrals $J_{ \pm}, T_{ \pm}$and $S_{ \pm}$, being quadratic in $p_{i}$, correspond here to generators of the hidden symmetries [1]. 
The ten generators (3.1)-(3.3) satisfy the following Poisson bracket relations of the $\mathfrak{s p}(4, \mathbb{R})$ algebra,

$$
\begin{aligned}
& \{D, H\}=H, \\
& \{D, K\}=-K, \quad\{K, H\}=2 D, \\
& \left\{J_{0}, J_{ \pm}\right\}=\mp i J_{ \pm}, \quad\left\{J_{-}, J_{+}\right\}=-2 i J_{0}, \\
& \left\{J_{0}, T_{ \pm}\right\}=\mp i T_{ \pm}, \quad\left\{J_{0}, S_{ \pm}\right\}=\mp i S_{ \pm}, \\
& \left\{H, S_{ \pm}\right\}=-2 J_{ \pm}, \quad\left\{H, J_{ \pm}\right\}=-T_{ \pm} \text {, } \\
& \left\{K, T_{ \pm}\right\}=2 J_{ \pm}, \quad\left\{K, J_{ \pm}\right\}=S_{ \pm}, \\
& \left\{D, T_{ \pm}\right\}=T_{ \pm}, \quad\left\{D, S_{ \pm}\right\}=-S_{ \pm}, \\
& \left\{S_{ \pm}, T_{\mp}\right\}=\mp 4 i\left(J_{0} \pm i D\right) \text {, } \\
& \left\{J_{ \pm}, T_{\mp}\right\}=2 H \text {, } \\
& \left\{J_{ \pm}, S_{\mp}\right\}=2 K .
\end{aligned}
$$

By including the first order generators (3.4) with redefinition $\xi_{ \pm}=m \chi_{ \pm}$, we supplement the algebra (3.5)-(3.12) with the Poisson bracket relations

$$
\begin{aligned}
& \left\{\xi_{ \pm}, p_{\mp}\right\}=2 m \\
& \left\{H, \xi_{ \pm}\right\}=-p_{ \pm}, \quad\left\{D, \xi_{ \pm}\right\}=-\frac{1}{2} \xi_{ \pm}, \quad\left\{J_{0}, \xi_{ \pm}\right\}=\mp \frac{i}{2} \xi_{ \pm}, \\
& \left\{K, p_{ \pm}\right\}=\xi_{ \pm}, \quad\left\{D, p_{ \pm}\right\}=\frac{1}{2} p_{ \pm}, \quad\left\{J_{0}, p_{ \pm}\right\}=\mp \frac{i}{2} p_{ \pm}, \\
& \left\{T_{ \pm}, \xi_{\mp}\right\}=-2 p_{ \pm}, \quad\left\{S_{ \pm}, p_{\mp}\right\}=2 \xi_{ \pm} \\
& \left\{J_{ \pm}, \xi_{\mp}\right\}=-\xi_{ \pm}, \quad\left\{J_{ \pm}, p_{\mp}\right\}=p_{ \pm} .
\end{aligned}
$$

The not displayed in (3.5)-(3.17) Poisson brackets are equal to zero. Relations (3.13)(3.17) correspond to the ideal sub-algebra generated by $\xi_{ \pm}$and $p_{ \pm}$, with mass $m$ playing a role of the central charge. This also is an ideal sub-algebra of the Schrödinger algebra $\mathfrak{s c h}(2)$ [56], generated by $H, D, K, p_{i}, \xi_{i}$ and $m$, that, in turn, is a sub-algebra of the complete Lie algebra (3.5)-(3.17).

Some remarkable properties of the presented symmetry algebra are the following.

- The algebraic relations (3.5) correspond to the dynamical $\mathfrak{s o}(2,1) \cong \mathfrak{s l}(2, \mathbb{R})$ conformal algebra. Its Casimir element is $D^{2}-H K=-J_{0}^{2}=-\frac{1}{4} p_{\varphi}^{2}$.

- Relations (3.6) correspond to another $\mathfrak{s l}(2, \mathbb{R})$ sub-algebra with Casimir element $-J_{0}^{2}+J_{+} J_{-}=D^{2}$.

- Each triplet of integrals $\left(J_{0}, T_{ \pm}\right),\left(J_{0}, S_{ \pm}\right),\left(p_{\varphi}, p_{ \pm}\right)$and $\left(p_{\varphi}, \xi_{ \pm}\right)$generate Euclidean sub-algebra $\mathfrak{e}(2)$. The corresponding Casimirs $T_{+} T_{-}, S_{+} S_{-}, p_{+} p_{-}$and $\xi_{+} \xi_{-}$are $H^{2}$, $K^{2}, 2 m H$ and $2 m K$.

- The two sets $\left(\ell_{+}^{(+)}=\frac{1}{2 \gamma} S_{+}, \ell_{-}^{(+)}=\frac{\gamma}{2} T_{-}, \ell_{0}^{(+)}=\frac{1}{2}\left(J_{0}+i D\right)\right)$ and $\left(\ell_{+}^{(-)}=\frac{\gamma}{2} T_{+}\right.$, $\left.\ell_{-}^{(-)}=\frac{1}{2 \gamma} S_{-}, \ell_{0}^{(-)}=\frac{1}{2}\left(J_{0}-i D\right)\right)$ generate the $\mathfrak{s u}(2) \oplus \mathfrak{s u}(2)$ sub-algebra, where $\gamma$ is a constant of dimension of squared length that is introduced to compensate the corresponding dimensions. The Casimir elements of these two $\mathfrak{s u}(2)$ sub-algebras are $C^{( \pm)}=\left(\ell_{0}^{( \pm)}\right)^{2}+\ell_{+}^{( \pm)} \ell_{-}^{( \pm)}=0$. Note that $\left(\ell_{0}^{(+)}\right)^{*}=\ell_{0}^{(-)},\left(\ell_{+}^{( \pm)}\right)^{*}=\ell_{-}^{(\mp)}$. 
- Each integral is an eigenstate of $D$ in the sense of the Poisson bracket relation $\{D, A\}=\lambda A:\left(\lambda=1: H, T_{ \pm}\right),\left(\lambda=0: D, J_{0}, J_{ \pm}\right),\left(\lambda=-1: K, S_{ \pm}\right),\left(\lambda=1 / 2: p_{ \pm}\right)$, $\left(\lambda=-1 / 2: \xi_{ \pm}\right)$.

- Analogously, each integral is an eigenstate of $J_{0}:\left(\lambda=1: J_{+}, T_{+}, S_{+}\right),(\lambda=0$ : $\left.J_{0}, H, D, K\right),\left(\lambda=-1: J_{-}, T_{-}, S_{-}\right),\left(\lambda=1 / 2: p_{+}, \xi_{+}\right),\left(\lambda=-1 / 2: p_{-}, \xi_{-}\right)$.

Introducing linear combinations $\mathcal{J}_{0}=\frac{1}{2}(H+K), \mathcal{J}_{1}=\frac{1}{2}(H-K)$, and denoting $\mathcal{J}_{2}=D$, $\mathcal{J}_{ \pm}=\mathcal{J}_{1} \pm i \mathcal{J}_{2}$, conformal algebra (3.5) can be presented in the form similar to (3.6), and its Casimir takes the form $-\mathcal{J}_{0}^{2}+\mathcal{J}_{+} \mathcal{J}_{-}=-J_{0}^{2}$. The generators $\mathcal{J}_{\mu}, \mu=0,1,2$, of the conformal algebra describe the upper sheet of the two-sheeted hyperboloid, that at $p_{\varphi}=0$ degenerates into the cone with $\mathcal{J}_{0} \geq 0$. The generators $J_{\mu}$ of the $\mathfrak{s l}(2, \mathbb{R})$ algebra (3.6) describe a one sheet hyperboloid that at $\mathcal{J}_{2}=D=0$ degenerates into a double cone with $J_{0}=2 p_{\varphi} \in \mathbb{R}$.

Using the Casimir of conformal algebra (3.5) and the explicit form of generators (3.1), one gets

$$
r^{2}(t)=\frac{2}{m}\left(H t^{2}+2 D t+K\right)=\frac{2 H}{m}\left(\left(t+\frac{D}{H}\right)^{2}+\frac{p_{\varphi}^{2}}{4 H^{2}}\right)
$$

from where we see that at the moment of time $t_{*}=-D / H$, the particle is in the "perihelion" of the trajectory, $r\left(t_{*}\right) \equiv r_{*}=p_{\varphi} / \sqrt{2 m H}$. On the other hand, from the angular and linear momenta integrals the straight line trajectory is reconstructed in polar coordinates,

$$
r(\varphi)=\frac{r_{*}}{\cos \left(\varphi-\varphi_{*}\right)}, \quad-\pi / 2 \leq \varphi-\varphi_{*} \leq \pi / 2 .
$$

By means of (3.18) and (3.19) we also find

$$
\varphi(t)=\arctan \left(\frac{1}{2 \rho}\left(t+\frac{D}{H}\right)\right)+\varphi_{*}, \quad \rho=\frac{p_{\varphi}}{2 H} .
$$

From $\chi_{i}$ and $p_{i}$ one can construct a sort of Laplace-Runge-Lentz vector,

$$
\chi_{i}^{\perp}=x_{i}-p_{i} \frac{x_{j} p_{j}}{p_{k} p_{k}}
$$

such that $\chi_{i}^{\perp} p_{i}=0$. This vector (with respect to $p_{\varphi}$ ) integral specifies the coordinates of the perihelion, $\chi_{1}^{\perp}=r_{*} \cos \left(\varphi_{*}\right)=x_{1}\left(\varphi_{*}\right), \chi_{2}^{\perp}=r_{*} \sin \left(\varphi_{*}\right)=x_{2}\left(\varphi_{*}\right)$. The components $\chi_{i}^{\perp}$, however, are not independent integrals since they satisfy $\chi_{1}^{\perp}=\frac{p_{\varphi}}{2 m H} p_{2}$ and $\chi_{2}^{\perp}=-\frac{p_{\varphi}}{2 m H} p_{1}$.

In the quantum case, it is convenient here to use the polar coordinates, in which the Hamiltonian operator is given by

$$
\hat{H}=-\frac{\hbar^{2}}{2 m}\left(\frac{1}{r} \frac{\partial}{\partial r}\left(r \frac{\partial}{\partial r}\right)+\frac{1}{r^{2}} \frac{\partial^{2}}{\partial \varphi}\right) .
$$

Its eigenstates and eigenvalues are

$$
\psi_{\kappa, l}^{ \pm}(r, \varphi)=\sqrt{\frac{\kappa}{2 \pi}} J_{l}(\kappa r) e^{ \pm i l \varphi}, \quad E=\frac{\hbar^{2} \kappa^{2}}{2 m}, \quad l=0,1, \ldots,
$$


where $J_{\beta}(\zeta)$ are the Bessel functions of the first kind. With respect to the inner product

$$
\left\langle\Psi_{1} \mid \Psi_{2}\right\rangle=\int_{0}^{\infty} r d r \int_{0}^{2 \pi} d \varphi \Psi_{1}^{*} \Psi_{2}
$$

eigenstates (3.23) satisfy the orthogonality relation $\left\langle\psi_{\kappa, l}^{ \pm} \mid \psi_{\kappa^{\prime}, l^{\prime}}^{\mp}\right\rangle=\delta_{l l^{\prime}} \delta\left(\kappa-\kappa^{\prime}\right)$, and due to the property of the Bessel functions $J_{-l}(\eta)=(-1)^{l} J_{l}(\eta)$, one has $\psi_{\kappa,-l}^{ \pm}=(-1)^{l} \psi_{\kappa, l}^{\mp}$.

The basic first order differential operators of the system in polar coordinates are

$$
\hat{p}_{r}=-i \hbar\left(\frac{\partial}{\partial r}+\frac{1}{2 r}\right), \quad \hat{p}_{\varphi}=-i \hbar \frac{\partial}{\partial \varphi},
$$

where $\hat{p}_{r}$ is symmetric with respect to (3.24), but not self-adjoint. We use them to construct the well-defined operators

$$
\hat{p}_{ \pm}=-i \hbar e^{ \pm i \varphi}\left[\frac{\partial}{\partial r} \pm i \frac{1}{r} \frac{\partial}{\partial \varphi}\right], \quad p_{+}^{\dagger}=\hat{p}_{-},
$$

which are the quantum version of the classical integrals $p_{ \pm}$. Their action on eigenstates can be found by using the recurrence relations $\frac{2 \beta}{\zeta} J_{\beta}(\zeta)=J_{\beta-1}(\zeta)+J_{\beta+1}(\zeta), 2 \frac{d}{d \zeta} J_{\zeta}(\zeta)=$ $J_{\beta-1}(\zeta)-J_{\beta+1}(\zeta)$, and we get

$$
\hat{p}_{ \pm} \psi_{\kappa, l}^{ \pm}(r, \varphi)=i \hbar \kappa \psi_{\kappa, l+1}^{ \pm}(r, \varphi), \quad \hat{p}_{ \pm} \psi_{\kappa, l}^{\mp}(r, \varphi)=-i \hbar \kappa \psi_{\kappa, l-1}^{\mp}(r, \varphi) .
$$

These relations show that the operators $\hat{p}_{ \pm}$change the angular momentum quantum number of the wave-function without changing the energy, that reflects the infinite degeneracy of the energy levels.

Quantum version of other integrals is obtained by the substitution $p_{ \pm} \rightarrow \hat{p}_{ \pm}$and using the Weyl (symmetric) ordering in (3.1)-(3.3). In the general case we have dynamical integrals $\hat{A}(t)$ which include the explicit dependence on time, $\frac{d}{d t} \hat{A}(t)=\frac{\partial}{\partial t} \hat{A}(t)+\frac{1}{i \hbar}[\hat{A}(t), \hat{H}]=0$. For them we have

$$
\hat{A}(t) \Psi(r, \varphi, t)=\left.e^{-\frac{i t \hat{H}}{\hbar}} \hat{A}\right|_{t=0} \Psi(r, \varphi, t=0),
$$

where $\Psi(r, \varphi, t)$ is a solution of the time dependent Schrödinger equation. In particular, the quantum generators of dilatations and special conformal transformations,

$$
\begin{aligned}
& \hat{D}=\frac{1}{4}\left(\hat{\chi}_{+} \hat{p}_{-}+\hat{p}_{+} \hat{\chi}_{-}\right)=-i \frac{\hbar}{2}\left(r \frac{\partial}{\partial r}+1\right)-\hat{H} t, \\
& \hat{K}=\frac{1}{2} m \hat{\chi}_{-} \hat{\chi}_{+}=\frac{1}{2} m r^{2}-2 \hat{D} t-\hat{H} t^{2},
\end{aligned}
$$

are examples of dynamical symmetry operators. Together with the Hamiltonian, they generate the quantum $\mathfrak{s l}(2, \mathbb{R})$ algebra

$$
[\hat{D}, \hat{H}]=i \hbar \hat{H}, \quad[\hat{D}, \hat{K}]=-i \hbar \hat{K}, \quad[\hat{K}, \hat{H}]=2 i \hbar \hat{D} .
$$

On a Hilbert subspace with fixed value of the quantum number $l=0,1, \ldots$, the eigenstates (3.23) correspond to an irreducible infinite dimensional representation of conformal $\mathfrak{s l}(2, \mathbb{R})$ algebra $(3.31)$ of the discrete type series $D_{j}^{+}$characterized by the Casimir operator 
value $-\hat{\mathcal{J}}_{0}^{2}+\hat{\mathcal{J}}_{1}^{2}+\hat{\mathcal{J}}_{2}^{2}=\hat{D}^{2}-\frac{1}{2}(\hat{K} \hat{H}+\hat{H} \hat{K})=-\hbar^{2} j(j-1)$ with $j=\frac{1}{2}(l+1)$, and eigenvalues of the compact generator $\hat{\mathcal{J}}_{0}$ to be $j+n, n=0,1, \ldots$ The present free particle's Hilbert space, in which the non-compact $\mathfrak{s l}(2, \mathbb{R})$ generator $\hat{\mathcal{J}}_{0}+\hat{\mathcal{J}}_{1}=\hat{H}$ is diagonal, corresponds to the so-called parabolic realization of $D_{j}^{+}$representation [63].

For quantum analog of the $\mathfrak{s l}(2, \mathbb{R})$ algebra (3.6), the Casimir operator is $\hat{J}_{0}\left(-\hat{J}_{0}+\hbar\right)+$ $\hat{J}_{+} \hat{J}_{-}=\hat{D}^{2}+\frac{1}{4} \hbar^{2}$. Operator $\hat{D}$ is self-adjoint with respect to the scalar product $(3.24)$, and at $t=0$ its eigenfunctions are $\Psi_{\lambda}(r)=r^{2 i \lambda-1} / \sqrt{2} \pi, \hat{D} \Psi_{\lambda}=\lambda \Psi_{\lambda}, \lambda \in \mathbb{R},\left\langle\Psi_{\lambda} \mid \Psi_{\lambda}^{\prime}\right\rangle=$ $\delta\left(\lambda-\lambda^{\prime}\right)$. One sees then that the quantum analog of the $\mathfrak{s l}(2, \mathbb{R})$ algebra (3.6) at fixed value of $\lambda$ corresponds here to the principal continuous series representation characterized by the Casimir invariant value $\hat{C}=\hbar^{2}\left(\lambda^{2}+1 / 4\right) \geq \hbar^{2} / 4$, in which the eigenvalues of the compact generator $\hat{J}_{0}$ are $j_{0}=\hbar j$ with $j=0, \pm 1, \pm 2, \ldots$ on the subspace with even values of $l$, and $j= \pm 1 / 2, \pm 3 / 2, \ldots$ on the subspace with odd values of $l$ [64].

The difference between representations generated by $\hat{\mathcal{J}}_{\mu}$ and $\hat{J}_{\mu}$ operators is coherent with the difference of the above-mentioned corresponding classical hyperboloids [63].

\subsection{The isotropic harmonic oscillator}

Here we consider some general properties of the harmonic oscillator in the Euclidean plane. First, we construct the symmetry generators and the symmetry algebra. In the next step, we use these integrals of motion to algebraically reproduce the orbit of the particle from the integrals of motion, and finally, we review the quantum picture of the model.

The classical Hamiltonian of the planar isotropic harmonic oscillator system

$$
H_{\mathrm{os}}=H_{1}+H_{2}=\frac{p_{r}^{2}}{2 m}+\frac{p_{\varphi}^{2}}{2 m r^{2}}+\frac{m \omega^{2}}{2} r^{2}
$$

can be understood in Cartesian coordinates $x_{j}, j=1,2$, as the sum of the two independent one-dimensional harmonic oscillator Hamiltonians $H_{j}=\frac{1}{2 m}\left(p_{j}^{2}+m^{2} \omega^{2} x_{j}^{2}\right)$ with the same frequencies and masses, while in the polar coordinates it can be considered as a two-dimensional generalization of the de Alfaro Fubini and Furlan conformal mechanics model [16].

The quantities

$$
a_{j}^{ \pm}=\frac{1}{\sqrt{2}} e^{\mp i \omega t}\left(\sqrt{m \omega} x_{j} \mp i \frac{p_{j}}{\sqrt{m \omega}}\right), \quad j=1,2,
$$

being classical analogs of the quantum ladder operators multiplied by $e^{\mp i \omega t}$, are the basic dynamical integrals expressed in Cartesian coordinates. Their linear combinations

$$
\begin{array}{ll}
b_{1}^{-}=\frac{1}{\sqrt{2}}\left(a_{1}^{-}-i a_{2}^{-}\right)=\frac{1}{2} e^{i(\omega t-\varphi)}\left(\sqrt{m \omega} r+\frac{p_{\varphi}}{\sqrt{m \omega} r}+i \frac{p_{r}}{\sqrt{m \omega}}\right), & b_{1}^{+}=\left(b_{1}^{-}\right)^{*}, \\
b_{2}^{-}=\frac{1}{\sqrt{2}}\left(a_{1}^{-}+i a_{2}^{-}\right)=\frac{1}{2} e^{i(\omega t+\varphi)}\left(\sqrt{m \omega} r-\frac{p_{\varphi}}{\sqrt{m \omega} r}+i \frac{p_{r}}{\sqrt{m \omega}}\right), & b_{2}^{+}=\left(b_{2}^{-}\right)^{*},
\end{array}
$$

are more convenient, however, when we work in the polar coordinates. They can be produced by a particular classical canonical transformation, or the corresponding unitary 
transformation at the quantum level, that we consider below. The ten second-order in these basic integrals symmetry generators are

$$
\begin{array}{rlrl}
\mathcal{J}_{0} & =\frac{1}{2} b_{j}^{+} b_{j}^{-}=\frac{1}{2 \omega} H_{\mathrm{os}}, & \mathcal{L}_{2}=\frac{1}{2}\left(b_{1}^{+} b_{1}^{-}-b_{2}^{+} b_{2}^{-}\right)=\frac{1}{2} p_{\varphi}, \quad \mathcal{L}_{ \pm}=b_{1}^{ \pm} b_{2}^{\mp}, \\
\mathcal{J}_{ \pm}=b_{1}^{ \pm} b_{2}^{ \pm}=\frac{1}{2}\left(\left(a_{1}^{ \pm}\right)^{2}+\left(a_{2}^{ \pm}\right)^{2}\right), & \mathcal{B}_{j}^{ \pm}=\left(b_{j}^{ \pm}\right)^{2} .
\end{array}
$$

Unlike the free particle case, only four integrals (3.36) here do not depend explicitly on time. The integrals (3.36) and (3.37) satisfy the following non-zero Poisson bracket relations

$$
\begin{array}{lll}
\left\{\mathcal{J}_{0}, \mathcal{J}_{ \pm}\right\}=\mp i \mathcal{J}_{ \pm}, & \left\{\mathcal{J}_{-}, \mathcal{J}_{+}\right\}=-2 i \mathcal{J}_{0}, & \\
\left\{\mathcal{L}_{2}, \mathcal{L}_{ \pm}\right\}=\mp i \mathcal{L}_{ \pm}, & \left\{\mathcal{L}_{+}, \mathcal{L}_{-}\right\}=-i 2 \mathcal{L}_{2}, & \\
\left\{\mathcal{J}_{ \pm}, \mathcal{L}_{\mp}\right\}= \pm i \mathcal{B}_{2}^{ \pm}, & \left\{\mathcal{J}_{ \pm}, \mathcal{L}_{ \pm}\right\}= \pm i \mathcal{B}_{1}^{ \pm} & \\
\left\{\mathcal{J}_{0}, \mathcal{B}_{a}^{ \pm}\right\}=\mp i \mathcal{B}_{a}^{ \pm}, & \left\{\mathcal{J}_{\mp}, \mathcal{B}_{2}^{ \pm}\right\}=\mp 2 i \mathcal{L}_{\mp}, & \left\{\mathcal{J}_{\mp}, \mathcal{B}_{1}^{ \pm}\right\}=\mp 2 i \mathcal{L}_{ \pm}, \\
\left\{\mathcal{L}_{2}, \mathcal{B}_{1}^{ \pm}\right\}=\mp i \mathcal{B}_{1}^{ \pm}, & \left\{\mathcal{L}_{2}, \mathcal{B}_{2}^{ \pm}= \pm i \mathcal{B}_{2}^{ \pm},\right. & \left\{\mathcal{L}_{ \pm}, \mathcal{B}_{1}^{\mp}\right\}= \pm 2 i \mathcal{J}_{\mp} \\
\left\{\mathcal{L}_{ \pm}, \mathcal{B}_{2}^{ \pm}\right\}=\mp 2 i \mathcal{J}_{ \pm}, & \left\{\mathcal{B}_{1}^{-}, \mathcal{B}_{1}^{+}\right\}=-4 i\left(\mathcal{J}_{0}+\mathcal{L}_{2}\right), & \left\{\mathcal{B}_{2}^{-}, \mathcal{B}_{2}^{+}\right\}=-4 i\left(\mathcal{J}_{0}-\mathcal{L}_{2}\right) .
\end{array}
$$

The brackets involving the basic integrals are

$$
\begin{array}{rlrl}
\left\{b_{i}^{-}, b_{j}^{+}\right\} & =-i \delta_{i j}, & \\
\left\{\mathcal{J}_{0}, b_{j}^{ \pm}\right\} & =\mp \frac{i}{2} b_{j}^{ \pm}, & \left\{\mathcal{J}_{\mp}, b_{1}^{ \pm}\right\}=\mp i b_{2}^{\mp}, & \left\{\mathcal{J}_{\mp}, b_{2}^{ \pm}\right\}=\mp i b_{1}^{\mp}, \\
\left\{\mathcal{B}_{i}^{ \pm}, b_{j}^{\mp}\right\} & = \pm 2 i \delta_{i j} b_{j}^{\mp}, & \left\{\mathcal{L}_{2}, b_{1}^{ \pm}\right\}=\mp \frac{i}{2} b_{1}^{ \pm}, & \left\{\mathcal{L}_{2}, b_{2}^{ \pm}\right\}= \pm \frac{i}{2} b_{2}^{ \pm}, \\
\left\{\mathcal{L}_{ \pm}, b_{1}^{\mp}\right\} & = \pm i b_{2}^{\mp}, & \left\{\mathcal{L}_{ \pm}, b_{2}^{ \pm}\right\}=\mp i b_{1}^{ \pm}, & \\
\left\{\mathcal{J}_{ \pm}, b_{j}^{ \pm}\right\} & =\left\{\mathcal{L}_{ \pm}, b_{1}^{ \pm}\right\}=\left\{\mathcal{L}_{ \pm}, b_{2}^{\mp}\right\}=0 . &
\end{array}
$$

Some properties of the Lie algebra (3.38)-(3.48) are the following.

- Dynamical integrals $b_{j}^{ \pm}$generate an ideal sub-algebra.

- Relations (3.38) correspond to the conformal $\mathfrak{s l}(2, \mathbb{R})$ symmetry. The Casimir is $-\mathcal{J}_{0}^{2}+\mathcal{J}_{+} \mathcal{J}_{-}=-\mathcal{L}_{2}^{2}$

- The not-depending explicitly on time integrals of motion $\mathcal{L}_{2}$ and $\mathcal{L}_{ \pm}=\mathcal{L}_{3} \pm i \mathcal{L}_{1}$ generate the $\mathfrak{s u}(2)$ algebra (3.39) with the Casimir invariant $\mathcal{L}_{1}^{2}+\mathcal{L}_{2}^{2}+\mathcal{L}_{3}^{2}=\mathcal{J}_{0}^{2}=\frac{1}{4 \omega^{2}} H_{\text {os }}^{2}$.

- Each triplet of integrals $\left(\mathcal{L}_{2}, \mathcal{B}_{j}^{-}\right),\left(\mathcal{L}_{2}, \mathcal{B}_{j}^{+}\right),\left(p_{\varphi}, b_{j}^{-}\right)$and $\left(p_{\varphi}, b_{j}^{+}\right)$generate Euclidean sub-algebra $\mathfrak{e}(2)$. The corresponding Casimirs $\mathcal{B}_{1}^{-} \mathcal{B}_{2}^{-}, \mathcal{B}_{1}^{+} \mathcal{B}_{2}^{+}, b_{1}^{-} b_{2}^{-}$, and $b_{1}^{+} b_{2}^{+}$are $\mathcal{J}_{-}^{2}$, $\mathcal{J}_{+}^{2}, \mathcal{J}_{-}$and $\mathcal{J}_{+}$, respectively.

- The sets of integrals $\left(\mathscr{J}_{ \pm}^{(+)}=\mathcal{B}_{1}^{ \pm} / \sqrt{2}, \mathscr{J}_{0}^{(+)}=\mathcal{J}_{0}+\mathcal{L}_{2}\right)$, and $\left(\mathscr{J}_{ \pm}^{(-)}=\mathcal{B}_{2}^{ \pm} / \sqrt{2}\right.$, $\left.\mathscr{J}_{0}^{(-)}=\mathcal{J}_{0}-\mathcal{L}_{2}\right)$, generate the $\mathfrak{s l}(2, \mathbb{R}) \oplus \mathfrak{s l}(2, \mathbb{R})$ algebra. Note that generators $\mathscr{J}_{0}^{ \pm}$ can be reinterpreted as the Landau problem's Hamiltonians in the symmetric gauge, with positive/negative magnetic field of the magnitude $B=\frac{2 c m \omega}{q}$, where $q$ and $c$ correspond to the electric charge of the particle and the speed of light [55]. 
- Each integral is an eigenstate of $i \mathcal{J}_{0}$ in the sense of $\left\{i \mathcal{J}_{0}, A\right\}=\lambda A:\left(\lambda= \pm 1: \mathcal{J}_{ \pm}, \mathcal{B}_{j}^{ \pm}\right)$, $\left(\lambda=0: \mathcal{J}_{0}, \mathcal{L}_{2}, \mathcal{L}_{ \pm}\right),\left(\lambda= \pm 1 / 2: b_{j}^{ \pm}\right)$.

- Analogously, each integral is an eigenstate of $i \mathcal{L}_{2}:\left(\lambda=0: \mathcal{L}_{2}, \mathcal{J}_{0}, \mathcal{J}_{ \pm}\right),(\lambda= \pm 1$ : $\left.\mathcal{L}_{ \pm}, \mathcal{B}_{j}^{ \pm}\right),\left(\lambda= \pm 1 / 2: b_{1}^{\mp}, b_{2}^{ \pm}\right)$.

Using the dynamical integrals $\mathcal{J}_{ \pm}$and integral $\mathcal{J}_{0}$, we define the generators of the NewtonHooke conformal symmetry [51-55]

$$
\begin{aligned}
& \mathcal{K}=\frac{1}{2 \omega}\left(\mathcal{J}_{+}+\mathcal{J}_{-}+2 \mathcal{J}_{0}\right)=\frac{1}{2} m r^{2} \cos (2 \omega t)-\frac{1}{2 \omega} r p_{r} \sin (2 \omega t)+\frac{1}{\omega^{2}} H_{\mathrm{os}} \sin ^{2}(\omega t), \\
& \mathcal{D}=\frac{1}{2 i}\left(\mathcal{J}_{-}-\mathcal{J}_{+}\right)=\frac{1}{2} r p_{r} \cos (2 \omega t)-\frac{1}{2 \omega}\left(H_{\mathrm{os}}-m \omega^{2} r^{2}\right) \sin (2 \omega t) .
\end{aligned}
$$

Together with $H_{\mathrm{os}}=2 \omega \mathcal{J}_{0}$ they satisfy the Poisson bracket relations

$$
\left\{\mathcal{D}, H_{\text {os }}\right\}=H_{\text {os }}-2 \omega^{2} \mathcal{K}, \quad\{\mathcal{D}, \mathcal{K}\}=-\mathcal{K}, \quad\left\{H_{\text {os }}, \mathcal{K}\right\}=2 \mathcal{D},
$$

and in the limit $\omega \rightarrow 0$ take the form of the free particle integrals (3.1). From here, we obtain

$$
r^{2}(t)=\frac{1}{m \omega^{2}}\left(H_{\mathrm{os}}+2 \omega \mathcal{D} \sin (2 \omega t)+\left(2 \omega^{2} \mathcal{K}-H_{\mathrm{os}}\right) \cos (2 \omega t)\right) .
$$

Taking into account the equivalent form $\mathcal{D}^{2}+\omega^{2} \mathcal{K}^{2}-\mathcal{K} H_{\mathrm{os}}=-\frac{1}{4} p_{\varphi}^{2}$ for the Casimir of the conformal $\mathfrak{s l}(2, \mathbb{R})$ symmetry, equation (3.52) allows us to find the radial turning points

$$
r_{ \pm}^{2}=\frac{H_{\mathrm{os}}}{m \omega^{2}}(1 \pm \delta), \quad \delta=\sqrt{1-\frac{\omega^{2} p_{\varphi}^{2}}{H_{\mathrm{os}}^{2}}},
$$

which also can be found directly from the Hamiltonian by applying the condition $p_{r}=0$.

On the other hand, by using the explicit form of the integrals $\mathcal{L}_{1}$ and $\mathcal{L}_{3}$ in polar coordinates,

$$
\begin{aligned}
& \mathcal{L}_{1}=\sin (2 \varphi)\left(\frac{1}{2 \omega} H_{\mathrm{os}}-\frac{1}{2 m \omega r^{2}} p_{\varphi}^{2}\right)-\cos (2 \varphi) \frac{1}{2 m \omega r} p_{r} p_{\varphi}, \\
& \mathcal{L}_{3}=\cos (2 \varphi)\left(\frac{1}{2 \omega} H_{\mathrm{os}}-\frac{1}{2 m \omega r^{2}} p_{\varphi}^{2}\right)+\sin (2 \varphi) \frac{1}{2 m \omega r} p_{r} p_{\varphi},
\end{aligned}
$$

one deduces the elliptic trajectory for the isotropic planar harmonic oscillator,

$$
r^{2}(\varphi)=\frac{r_{0}^{2}}{1+\delta \cos \left(2\left(\varphi-\varphi_{*}\right)\right)}, \quad r_{0}^{2}=\frac{p_{\varphi}^{2}}{m H_{\mathrm{os}}},
$$

where $\varphi=\varphi_{*}$ corresponds to the angular position of one of the two "perihelia" of the trajectory. Eq. (3.56) has a form of elliptic trajectory in Kepler problem but with $r(\varphi)$ and $\varphi$ there changed for $r^{2}(\varphi)$ and $2 \varphi$ here. By means of (3.52) and (3.56) we also get

$$
\varphi(t)=\arctan \left(\frac{r_{+}}{r_{-}} \tan \left(\omega\left(t-t_{*}\right)\right)\right)+\varphi_{*} .
$$

where $t_{*}$ indicates the moment of time when the particle is in the corresponding perihelion.

Now let's take a look at the quantum case. We first consider the Cartesian coordinates representation, then we present the picture in the polar coordinates representation, and finally we show how these two representations are related to each other by a unitary transformation. 
Cartesian coordinates representation. The quantum versions of (3.33) (at $t=0$ ) are given by

$$
\hat{a}_{i}^{ \pm}=\sqrt{\frac{m \omega}{2 \hbar}}\left(x_{i} \mp \frac{\hbar}{m \omega} \frac{\partial}{\partial x_{i}}\right), \quad\left[\hat{a}_{i}^{-}, \hat{a}_{j}^{+}\right]=1 .
$$

In terms of them, we construct the set of operators (no summation in the repeated index),

$$
\begin{array}{rlrl}
\hat{\mathcal{J}}_{0}^{i} & =\frac{1}{2 \omega \hbar} \hat{H}_{i}=\frac{1}{4}\left(\hat{a}_{i}^{+} \hat{a}_{i}^{-}+\hat{a}_{i}^{-} \hat{a}_{i}^{+}\right), & \hat{\mathcal{J}}_{ \pm}^{i}=\frac{1}{2}\left(\hat{a}_{i}^{ \pm}\right)^{2}, \quad i=1,2, \\
\hat{\mathcal{L}}_{1}=\frac{1}{2}\left(\hat{a}_{1}^{+} \hat{a}_{2}^{-}+\hat{a}_{2}^{+} \hat{a}_{1}^{-}\right), & \hat{\mathcal{L}}_{2}=\frac{i}{2}\left(\hat{a}_{1}^{+} \hat{a}_{2}^{-}-\hat{a}_{2}^{+} \hat{a}_{1}^{-}\right)=\frac{1}{2 \hbar} \hat{p}_{\varphi}, \\
\hat{\mathcal{L}}_{3}=\frac{1}{2}\left(\hat{a}_{1}^{+} \hat{a}_{1}^{-}-\hat{a}_{2}^{+} \hat{a}_{2}^{-}\right)=\hat{\mathcal{J}}_{0}^{1}-\hat{\mathcal{J}}_{0}^{2}, & \hat{\mathcal{A}}_{ \pm}=\hat{a}_{1}^{ \pm} \hat{a}_{2}^{ \pm} .
\end{array}
$$

These ten operators satisfy the Lie algebra which (up to a unitary transformation) corresponds to the quantum version of the classical algebra (3.38)-(3.48). In this representation, the set of physical eigenstates and the spectrum of the system are

$$
\begin{aligned}
\psi_{n_{1}, n_{2}}\left(x_{1}, x_{2}\right) & =\psi_{n_{1}}\left(x_{1}\right) \psi_{n_{2}}\left(x_{2}\right), \quad E_{n_{1}, n_{2}}=\hbar\left(n_{1}+n_{2}+1\right), \quad n_{1,2}=0,1, \ldots \\
\psi_{n_{i}}\left(x_{i}\right) & =\frac{1}{\sqrt{2^{n} n !}}\left(\frac{m \omega}{\pi \hbar}\right)^{\frac{1}{4}} H_{n_{i}}\left(\sqrt{\frac{m \omega}{\hbar}} x_{i}\right) e^{-\frac{m \omega}{2 \hbar} x_{i}^{2}}
\end{aligned}
$$

where $H_{n_{i}}$ is the Hermite polynomial of order $n_{i}$. These wave-functions diagonalize simultaneously the operators $\hat{\mathcal{J}}_{0}^{i}, i=1,2$, and, as a consequence, $\hat{H}_{\text {os }}$ and $\hat{\mathcal{L}}_{3}$.

Polar coordinates representation. The quantum versions of the classical dynamical integrals (3.34), (3.35), (3.36) at $t=0$, and of the integrals (3.37) are

$$
\begin{aligned}
& \hat{b}_{1}^{-}=\frac{1}{\sqrt{2}}\left(\hat{a}_{1}^{-}-i \hat{a}_{2}^{-}\right)=\frac{1}{2} e^{-i \varphi} \sqrt{\frac{m \omega}{\hbar}}\left(r+\frac{\hbar}{m \omega}\left(\frac{\partial}{\partial r}-\frac{i}{r} \frac{\partial}{\partial \varphi}\right)\right), \quad \hat{b}_{1}^{+}=\left(\hat{b}_{1}^{-}\right)^{\dagger}, \\
& \hat{b}_{2}^{-}=\frac{1}{\sqrt{2}}\left(a_{1}^{-}+i a_{2}^{-}\right)=\frac{1}{2} e^{-i \varphi} \sqrt{\frac{m \omega}{\hbar}}\left(r+\frac{\hbar}{m \omega}\left(\frac{\partial}{\partial r}+\frac{i}{r} \frac{\partial}{\partial \varphi}\right)\right), \quad \hat{b}_{2}^{+}=\left(\hat{b}_{2}^{-}\right)^{\dagger}, \\
& \hat{\mathcal{J}}_{0}=\frac{1}{2 \hbar \omega} \hat{H}_{\mathrm{os}}=\frac{1}{4}\left(\left[\hat{b}_{1}^{+}, \hat{b}_{1}^{-}\right]_{+}+\left[\hat{b}_{2}^{+}, \hat{b}_{2}^{-}\right]_{+}\right), \quad \hat{\mathcal{L}}_{2}=\frac{1}{2}\left(\hat{b}_{1}^{+} \hat{b}_{1}^{-}-\hat{b}_{2}^{+} \hat{b}_{2}^{-}\right)=\frac{1}{2 \hbar} \hat{p}_{\varphi}, \\
& \hat{\mathcal{L}}_{ \pm}=\hat{b}_{1}^{ \pm} \hat{b}_{2}^{\mp}=\hat{\mathcal{L}}_{3} \pm i \hat{\mathcal{L}}_{1}=\frac{m \omega}{4 \hbar} e^{ \pm 2 i \varphi}\left(r^{2}+\frac{\hbar^{2}}{m^{2} \omega^{2}}\left(\frac{1}{r^{2}} \frac{\partial^{2}}{\partial \varphi^{2}}-\frac{\partial^{2}}{\partial r^{2}} \pm i \frac{2}{r} \frac{\partial^{2}}{\partial r \partial \varphi}\right)\right), \\
& \hat{\mathcal{J}}_{ \pm}=\hat{b}_{1}^{ \pm} \hat{b}_{2}^{ \pm}=-\frac{m \omega}{4 \hbar}\left(\hat{H}_{\mathrm{os}}-m \omega^{2} r^{2} \pm \hbar \omega\left(r \frac{\partial}{\partial r}+1\right)\right), \quad \hat{\mathcal{B}}_{j}^{ \pm}=\left(\hat{b}_{j}^{ \pm}\right)^{2},
\end{aligned}
$$

where $[,]_{+}$is the anti-commutator of the operators. In this representation we diagonalize simultaneously $\hat{H}_{\mathrm{oS}}=2 \hbar \omega \hat{\mathcal{J}}_{0}$ and $\hat{p}_{\varphi}=2 \hbar \hat{\mathcal{L}}_{2}$. The eigenstates and spectrum are

$$
\begin{array}{rlrl}
\psi_{n_{r}, l}^{ \pm}(r, \varphi) & =\left(\frac{m \omega}{\hbar}\right)^{\frac{1}{2}} \sqrt{\frac{n_{r} !}{2 \pi \Gamma\left(n_{r}+l+1\right)}} \zeta^{l} L_{n_{r}}^{(l)}\left(\zeta^{2}\right) e^{-\frac{\zeta^{2}}{2} \pm i l \varphi}, \quad \zeta & =\sqrt{\frac{m \omega}{\hbar}} r \\
E_{n, l} & =\hbar \omega\left(2 n_{r}+l+1\right), & n_{r}, l & =0,1, \ldots,
\end{array}
$$

where $L_{n_{r}}^{(l)}$ are the Laguerre polynomials. 
Unitary transformation. The two representations corresponding to Cartesian and polar coordinates can be related by the unitary operator

$$
\hat{U}=\exp \left(-i \frac{2 \pi}{3} \frac{1}{\sqrt{3}}\left(\hat{\mathcal{L}}_{1}+\hat{\mathcal{L}}_{2}+\hat{\mathcal{L}}_{3}\right)\right)
$$

which produces the rotation of the $\mathfrak{s u}(2)$ generators in the "ambient three-dimensional space" for the angle $-2 \pi / 3$,

$$
\hat{U} \hat{\mathcal{L}}_{1} \hat{U}^{\dagger}=\hat{\mathcal{L}}_{3}, \quad \hat{U} \hat{\mathcal{L}}_{2} \hat{U}^{\dagger}=\hat{\mathcal{L}}_{1}, \quad \hat{U} \hat{\mathcal{L}}_{3} \hat{U}^{\dagger}=\hat{\mathcal{L}}_{2} .
$$

Then, from $\hat{\mathcal{L}}_{3} \psi_{n_{1}, n_{2}}=\frac{n_{1}-n_{2}}{2} \psi_{n_{1}, n_{2}}$ we obtain $\hat{\mathcal{L}}_{2} \hat{U} \psi_{n_{1}, n_{2}}=\frac{n_{1}-n_{2}}{2} \hat{U} \psi_{n_{1}, n_{2}}$, and $\hat{U} \psi_{n_{1}, n_{2}}$ are the states that diagonalize the Hamiltonian $\hat{H}_{\mathrm{os}}=2 \hbar \omega \hat{\mathcal{J}}_{0}$ and the operator $\hat{p}_{\varphi}=2 \hbar \hat{\mathcal{L}}_{2}$ in the polar coordinates representation. Acting on operators $\hat{a}_{i}^{ \pm}$, the unitary transformation produces

$$
\hat{U} \hat{a}_{j}^{ \pm} \hat{U}^{\dagger}=e^{ \pm i \frac{\pi}{4}} \hat{b}_{j}^{ \pm}
$$

that corresponds to the spinor nature of the basic integrals with respect to the action of the $\mathfrak{s u}(2)$ generators in the ambient three-dimensional space.

In the next section we show how these two different physical systems, the free particle and harmonic oscillator in $\mathbb{R}^{2}$, are related to each other by the conformal bridge transformation [55]. This will serve as a precedent for the procedure that we will use to extract all the information for a system with the harmonic potential in conical geometry from the free dynamics on the same geometric background.

\section{The conformal bridge transformation}

In the general case, a mechanical system is governed by a symmetry algebra which encodes its peculiarities. At the classical level, these symmetries can be related with the geometric properties of the trajectory, and at the quantum level they encode the information related with the energy spectrum.

According to Dirac [65], a given symmetry algebra (up to isomorphisms) can represent different mechanical systems. In this section we show how the two forms of dynamics associated with the conformal algebra $\mathfrak{s o}(2,1) \cong \mathfrak{s l}(2, \mathbb{R})$ are related to each other by means of a particular mapping, the so-called conformal bridge transformation [55]. This also will allow us to establish the interesting relation between the corresponding symmetry sub-algebras of the free particle and harmonic oscillator systems and understand the change of nature of some of them due to a non-unitarity of the conformal bridge transformation.

Consider the classical $\mathfrak{s o}(2,1)$ algebra

$$
\{D, H\}=H, \quad\{D, K\}=-K, \quad\{K, H\}=2 D,
$$

without specifying the form of the generators themselves. Now, let us introduce the following complex linear combinations of them,

$$
\mathcal{J}_{0}=\frac{1}{2}\left(\omega^{-1} H+\omega K\right), \quad \mathcal{J}_{ \pm}=-\frac{1}{2 \omega}\left(H-\omega^{2} K \pm i 2 \omega D\right)
$$


where $\omega$ is a constant that is introduced to compensate the dimensions of the generators. These new complex quantities satisfy the classical $\mathfrak{s l}(2, \mathbb{R})$ algebra

$$
\left\{\mathcal{J}_{0}, \mathcal{J}_{ \pm}\right\}=\mp i \mathcal{J}_{ \pm}, \quad\left\{\mathcal{J}_{-}, \mathcal{J}_{+}\right\}=-2 i \mathcal{J}_{0} .
$$

Independently, both algebraic structures can represent different physical systems. In the case where $H$ is the Hamiltonian of a certain asymptotically free model with conformal symmetry (the free particle, for example), we have that $K$ and $D$ are dynamical integrals that explicitly depend on $t$. From here one has

$$
\begin{array}{ll}
K=T_{H}(t)\left(K_{0}\right), & K_{0}=T_{H}(-t)(K)=\left.K\right|_{t=0}, \\
D=T_{H}(t)\left(D_{0}\right), & D_{0}=T_{H}(-t)(D)=\left.D\right|_{t=0},
\end{array}
$$

where $T_{H}( \pm t)$ denotes a Hamiltonian flow, which is a canonical transformation generated by the Hamiltonian itself. The flow generated by a phase space function $F$ is

$$
\exp (\gamma F) \star f(q, p):=f(q, p)+\sum_{n=1}^{\infty} \frac{\gamma^{n}}{n !}\left\{F,\left\{\ldots,\{F, f \underbrace{\} \ldots\}\}}_{n}=: T_{F}(\gamma)(f) .\right.\right.
$$

In the same way, the compact generator $2 \omega \mathcal{J}_{0}$ (ignoring the dependence on $t$ of $F$ in the definition (4.2)) can be interpreted as the Hamiltonian of a harmonically trapped system (the harmonic oscillator, for example) with frequency $\omega$, and the quantities $\mathcal{J}_{ \pm}$are its dynamical integrals that satisfy

$$
\mathcal{J}_{ \pm}=T_{2 \omega \mathcal{J}_{0}}(\tau)\left(\left.\mathcal{J}_{ \pm}\right|_{t=0}\right),\left.\quad \mathcal{J}_{ \pm}\right|_{t=0}=T_{2 \omega \mathcal{J}_{0}}(-\tau)\left(\mathcal{J}_{ \pm}\right)
$$

Both forms of dynamics are related to each other by the complex canonical transformation

$$
\mathscr{T}(\tau, \beta, \delta, \gamma, t)=T_{2 \omega \mathcal{J}_{0}}(\tau) \circ T_{\beta \delta \gamma} \circ T_{H}(-t),
$$

where

$$
\begin{gathered}
T_{\beta \delta \gamma}:=T_{K_{0}}(\beta) \circ T_{H}(\delta) \circ T_{D_{0}}(\gamma)=T_{K_{0}}(\delta) \circ T_{D_{0}}(\gamma) \circ T_{H}(2 \delta), \\
\delta=\frac{i}{2 \omega}, \quad \beta=-i \omega, \quad \gamma=-\ln 2 .
\end{gathered}
$$

In this composition, the first transformation $T_{H}(-t)$ removes the $t$ dependence in the dynamical integrals $D$ and $K$. The second transformation relates these $t=0$ generators with the generators of the $\mathfrak{s l}(2, \mathbb{R})$ algebra $\mathcal{J}_{0}$ and $\mathcal{J}_{ \pm}$taken at $\tau=0$. The last transformation $T_{2 \omega \mathcal{J}_{0}}(\tau)$ restores the $\tau$ dependence. Explicitly one has

$$
\begin{array}{ll}
\mathscr{T}(\tau, \beta, \delta, \gamma, t)(H)=-\omega \mathcal{J}_{-}, & \mathscr{T}(\tau, \beta, \delta, \gamma, t)(D)=-i \mathcal{J}_{0}, \\
\mathscr{T}(\tau, \beta, \delta, \gamma, t)(K) & =\frac{1}{\omega} \mathcal{J}_{+} .
\end{array}
$$

The corresponding inverse transformation is given by

$$
\begin{aligned}
(\mathscr{T}(\tau, \beta, \delta, \gamma, t))^{-1} & =T_{H}(t) \circ\left(T_{\beta \delta \gamma}\right)^{-1} \circ T_{2 \omega \mathcal{J}_{0}}(-\tau), \\
\left(T_{\beta \delta \gamma}\right)^{-1} & =T_{D_{0}}(-\gamma) \circ T_{H}(-\delta) \circ T_{K_{0}}(-\beta) .
\end{aligned}
$$




\begin{tabular}{|c|c|}
\hline Free Particle & Harmonic Oscillator \\
\hline $\mathfrak{s o}(2,1):(H, D, K)$ & $\mathfrak{s l}(2, \mathbb{R}):\left(\mathcal{J}_{0}, \mathcal{J}_{ \pm}\right)$ \\
$\mathfrak{s l}(2, \mathbb{R}):\left(J_{0}, J_{ \pm}\right)$ & $\mathfrak{s u}(2):\left(\mathcal{L}_{2}, \mathcal{L}_{ \pm}\right)$ \\
$\mathfrak{e}_{2}:\left(J_{0}, T_{ \pm}\right)$ & $\mathfrak{e}_{2}:\left(\mathcal{L}_{2}, \mathcal{B}_{j}^{-}\right)$ \\
$\mathfrak{e}_{2}:\left(J_{0}, S_{ \pm}\right)$ & $\mathfrak{e}_{2}:\left(\mathcal{L}_{2}, \mathcal{B}_{j}^{+}\right)$ \\
$\mathfrak{e}_{2}:\left(p_{\varphi}, p_{ \pm}\right)$ & $\mathfrak{e}_{2}:\left(\mathcal{L}_{2}, b_{j}^{-}\right)$ \\
$\mathfrak{e}_{2}:\left(p_{\varphi}, \xi_{ \pm}\right)$ & $\mathfrak{e}_{2}:\left(\mathcal{L}_{2}, b_{j}^{+}\right)$ \\
$\mathfrak{s u}(2) \oplus \mathfrak{s u}(2):\left(\ell_{0}^{( \pm)}, \ell_{ \pm}^{( \pm)}\right)$ & $\mathfrak{s l}(2, \mathbb{R}) \oplus \mathfrak{s l}(2, \mathbb{R}):\left(\mathscr{J}_{0}^{( \pm)}, \mathscr{J}_{ \pm}^{( \pm)}\right)$ \\
\hline
\end{tabular}

Table 1. Correspondence between some subalgebras and their generators.

This transformation is a generalization of the classical version of the quantum conformal bridge transformation introduced in [55], and corresponds to an automorphism of algebra since it relates the Wick rotated non-compact generator $i D$ and compact generator $\mathcal{J}_{0}$ to each other, the real-valued non-compact generators $H$ and $K$ with the non-compact complex-valued generators $-\omega \mathcal{J}_{-}$and $\frac{1}{\omega} \mathcal{J}_{+}$, respectively.

In the particular case of the free particle in the Euclidean space the generators of the $\mathfrak{s o}(2,1)$ algebra are specified in $(3.1)$. Then the generators of the $\mathfrak{s l}(2, \mathbb{R})$ algebra resulting from applying the transformation (4.8) are given by the planar harmonic oscillator generators $\mathcal{J}_{0}$ and $\mathcal{J}_{ \pm}$defined in (3.36)-(3.37). The transformation also allows us to map the remaining symmetry generators of the free particle system into the corresponding generators of the harmonic oscillator. In particular, by direct application of the transformation to the generators $p_{ \pm}$and $\xi_{ \pm}$we get

$$
\begin{array}{llrl}
\mathscr{T}(\tau, \beta, \delta, \gamma, t)\left(p_{-}\right) & =-i \sqrt{2 m \omega} b_{1}^{-}, & \mathscr{T}(\tau, \beta, \delta, \gamma, t)\left(p_{+}\right) & =-i \sqrt{2 m \omega} b_{2}^{-}, \\
\mathscr{T}(\tau, \beta, \delta, \gamma, t)\left(\xi_{+}\right) & =\sqrt{\frac{2 m}{\omega}} b_{1}^{+}, & \mathscr{T}(\tau, \beta, \delta, \gamma, t)\left(\xi_{-}\right) & =\sqrt{\frac{2 m}{\omega}} b_{2}^{+},
\end{array}
$$

where the relations (3.44)-(3.48) were used. In the same vein, we list the effects of the transformation on the remaining second order generators,

$$
\begin{array}{rlrl}
\mathscr{T}(\tau, \beta, \delta, \gamma, t)\left(J_{0}\right) & =\mathcal{L}_{2}, & & \mathscr{T}(\tau, \beta, \delta, \gamma, t)\left(J_{ \pm}\right)=-i \mathcal{L}_{ \pm}, \\
\mathscr{T}(\tau, \beta, \delta, \gamma, t)\left(S_{+}\right)=\frac{1}{\omega} \mathcal{B}_{1}^{+}, & & \mathscr{T}(\tau, \beta, \delta, \gamma, t)\left(T_{-}\right)=-\omega \mathcal{B}_{1}^{-}, \\
\mathscr{T}(\tau, \beta, \delta, \gamma, t)\left(S_{-}\right)=\frac{1}{\omega} \mathcal{B}_{2}^{+}, & & \mathscr{T}(\tau, \beta, \delta, \gamma, t)\left(T_{+}\right)=-\omega \mathcal{B}_{2}^{-} .
\end{array}
$$

In table 1 we summarize the correspondence between generators and some subalgebras in both systems.

Note that the quantity $J_{0}=\mathcal{L}_{2}$ is the only object which is invariant under the transformation. This happens because the angular momentum is the only conserved quantity which Poisson commutes with all the $\mathfrak{s l}(2, \mathbb{R})$ conformal symmetry generators of the free particle. On the other hand, the second $\mathfrak{s l}(2, \mathbb{R})$ subalgebra is changed for the $\mathfrak{s u}(2)$ symmetry algebra after the transformation, and this is due to the imaginary unit appearing in 
the second equation in (4.17). In the same vein, the two copies of the $\mathfrak{s u}(2)$ algebra of the free particle with generators related by the complex conjugation are mapped into the two copies of the $\mathfrak{s l}(2, \mathbb{R})$ algebra associated with the Landau problem due to the appearance of the minus sign on the right hand side in the transformations of $T_{ \pm}$in (4.18) and (4.19).

The quantum analogue of the conformal bridge transformation corresponds to the non-unitary transformation produced by the operators

$$
\begin{aligned}
\hat{\mathfrak{S}}(t, \tau) & =e^{-\frac{i}{\hbar} 2 \omega \hat{\mathcal{J}}_{0} \tau} e^{-\frac{\omega}{\hbar} \hat{K}_{0}} e^{\frac{\hat{H}}{2 \hbar \omega}} e^{\frac{i}{\hbar} \ln (2) \hat{D}_{0}} e^{\frac{i}{\hbar} \hat{H} t} \\
\hat{\mathfrak{S}}^{-1}(t, \tau) & =e^{-\frac{i}{\hbar} \hat{H} t} e^{-\frac{i}{\hbar} \ln (2) \hat{D}_{0}} e^{-\frac{\hat{H}}{2 \hbar \omega}} e^{\frac{\omega}{\hbar} \hat{K}_{0}} e^{\frac{i}{\hbar} 2 \omega \hat{\mathcal{J}}_{0} \tau} .
\end{aligned}
$$

In this context, the Hamiltonian flux is changed by employing the Baker-CampbellHausdorff formula, and in correspondence with the established relation between the two-dimensional free particle and the planar isotropic harmonic oscillator in the Euclidean space, the classical relations (4.11)-(4.12) and (4.15)-(4.19) are preserved at the quantum level. ${ }^{1}$

In what concerns to eigenstates, in the general case the transformation implies that

$$
\begin{array}{lll}
\hat{D}|\lambda\rangle=i \hbar \lambda|\lambda\rangle & \Rightarrow \quad \hat{\mathcal{J}}_{0}(\hat{\mathfrak{S}}|\lambda\rangle)=\lambda \hat{\mathfrak{S}}|\lambda\rangle \\
\hat{H}|E\rangle=E|E\rangle \quad \Rightarrow \quad \hat{\mathcal{J}}_{-}(\hat{\mathfrak{S}}|E\rangle)=-\frac{E}{\hbar \omega} \hat{\mathfrak{S}}|E\rangle
\end{array}
$$

This means that the formal eigenstates of the dilatation operator with imaginary eigenvalue are mapped to the energy eigenstates of the harmonically confined system, while the asymptotic plane wave eigenstates of the asymptotically free Hamiltonian $\hat{H}$ correspond to coherent states of the system with the additional harmonic potential term, which, in turn, are eigenstates of the quadratic lowering operator. Note that to have the physically acceptable solutions, the states $\hat{\mathfrak{S}}|\lambda\rangle$ must be normalizable, and to ensure this, the three requirements must be met. First, the series

$$
e^{\frac{\hat{H}}{2 \hbar \omega}}|\lambda\rangle=\sum_{n=0}^{\infty} \frac{1}{n !(2 \hbar \omega)^{n}}(\hat{H})^{n}|\lambda\rangle
$$

has to reduce to a finite number of terms, i.e., $|\lambda\rangle$ should be the Jordan states $^{2}$ of $\hat{H}$ corresponding to zero energy [55]. Second, the function $\langle\boldsymbol{r} \mid \lambda\rangle$ must not have singularities in the corresponding operators domain. And finally, these functions must be single-valued with respect to the angular coordinate.

\footnotetext{
${ }^{1}$ We have considered dimensionless operators for the harmonic oscillator at the quantum level in the previous section. To recover these generators by the conformal bridge transformation we must compensate the multiplicative constants that appear in the quantum versions of the above-mentioned relationships. The relations involving the momenta operators linearly (quadratically) are multiplied by $\hbar^{1 / 2}(\hbar)$. This is taken into account in equations (4.20), (4.21).

${ }^{2}$ The Jordan states are given by wave functions that satisfy $P(\hat{H}) \Omega_{\lambda}=\psi_{\lambda}$, where $\hat{H} \psi_{\lambda}=\lambda \psi_{\lambda}$ and $P(\eta)$ represents a polynomial $[66,67]$. Here we consider Jordan states satisfying the relations $(\hat{H})^{\ell} \Omega_{\lambda}=\lambda \psi_{\lambda}$ with $\lambda=0$ for a certain natural $\ell$.
} 
In conclusion of this section, let us apply the inverse conformal bridge (similarity) transformation to the unitary operator (3.71). This yields us the non-unitary operator

$$
\hat{W}=\exp \left(-i \frac{2 \pi}{3} \frac{1}{\sqrt{3} \hbar}\left(\hat{J}_{0}+i\left(\hat{J}_{1}-i \hat{J}_{2}\right)\right)\right),
$$

and from equations (3.73) one obtains the transformation relations

$$
\begin{array}{rlrl}
\hat{W}\left(\hat{\xi}_{1}\right) \hat{W}^{-1} & =\frac{1}{\sqrt{2}} e^{i \frac{\pi}{4}} \hat{\xi}_{+}, & \hat{W}\left(\hat{\xi}_{2}\right) \hat{W}^{-1} & =\frac{1}{\sqrt{2}} e^{i \frac{\pi}{4}} \hat{\xi}_{-}, \\
\hat{W}\left(\hat{p}_{1}\right) \hat{W}^{-1}=\frac{1}{\sqrt{2}} e^{-i \frac{\pi}{4}} \hat{p}_{+}, & \hat{W}\left(\hat{p}_{2}\right) \hat{W}^{-1}=\frac{1}{\sqrt{2}} e^{-i \frac{\pi}{4}} \hat{p}_{-} .
\end{array}
$$

Equations (4.26), (4.27) provide us with the similarity transform of the Cartesian operators $\hat{\xi}_{j}$ and $\hat{p}_{j}$ into the complex operators $\hat{\xi}_{ \pm}$and $\hat{p}_{ \pm}$used in the polar coordinates representation for the quantum free particle. The conformal symmetry generators of the free particle, $\hat{H}$, $\hat{D}$ and $\hat{K}$, commute with the operator (4.25).

In the next section we finally pass over to the study of the free particle dynamics in conical geometry. Since the system possesses the conformal $\mathfrak{s t}(2, \mathbb{R})$ and rotational symmetries for any value of $\alpha$, then it will be possible to apply the conformal bridge transformation to analyze the dynamics of the harmonic oscillator in the same geometry.

\section{Free motion in a cosmic string background}

As we have shown in section 2, the study of the dynamics in a cosmic string background is analogous to analyzing the motion of a particle in conical geometry. A classical system in this space is governed by the action

$$
I=\int L d t, \quad L=\frac{m}{2} g_{i j} \frac{d x_{i}}{d t} \frac{d x_{j}}{d t}-V(\boldsymbol{r})=\frac{m}{2}\left(\alpha^{2} \dot{r}^{2}+r^{2} \dot{\varphi}^{2}\right)-V(\boldsymbol{r}) .
$$

In this section, we study the case of the free motion $(V(\boldsymbol{r})=0)$ from the perspective of its symmetries. We will show that when $\alpha$ is a rational number, it is possible to construct higher-order globally well-defined classical integrals of motion that can be identified as generators of hidden symmetries [1]. Then we study the system at the quantum level, and show that the only cases in which these conserved quantities can be promoted to the well-defined symmetry operators correspond to integer values of $\alpha$. Thus, we reveal here a kind of a quantum anomaly in the case of rational, non-integer values of $\alpha$.

\subsection{Classical case}

The classical dynamics of a free particle in a conical geometry is governed by the Hamiltonian

$$
H^{(\alpha)}=\frac{1}{2 m}\left(\frac{p_{r}^{2}}{\alpha^{2}}+\frac{p_{\varphi}^{2}}{r^{2}}\right) .
$$

Remarkably, the canonical transformation

$$
r \rightarrow \alpha r, \quad p_{r} \rightarrow \frac{p_{r}}{\alpha}, \quad \varphi \rightarrow \frac{\varphi}{\alpha}, \quad p_{\varphi} \rightarrow \alpha p_{\varphi},
$$


applied to the Hamiltonian of the Euclidean free particle gives us the Hamiltonian (5.2). Also note that when we apply this transformation to the usual Cartesian coordinates we get the "regularized" Cartesian coordinates (2.10). From the analysis of section 2 related to those coordinates it is clear that the canonical transformation (5.3) is welldefined only locally.

In spite of the indicated deficiency, we can use the canonical transformation (5.3) to reconstruct the solutions of the equations of motion of the system (5.2). The trajectory equation, as well as the time dependence of the radial and angular variables are immediately obtained from the corresponding relations (3.18), (3.19) and (3.20) for the free motion in the plane, and are given by

$$
\begin{aligned}
r(\varphi) & =\frac{r_{*}}{\cos \left(\left(\varphi-\varphi_{*}\right) / \alpha\right)}, & r_{*} & =\frac{p_{\varphi}}{\sqrt{2 m H^{(\alpha)}}}, \quad-\frac{\pi}{2} \alpha \leq \varphi-\varphi_{*} \leq \frac{\pi}{2} \alpha . \\
r^{2}(t) & =\frac{2}{\alpha^{2} m}\left(H^{(\alpha)} t^{2}+2 D t+K\right), & \varphi(t) & =\alpha \arctan \left(\frac{1}{2 \rho}\left(t+\frac{D}{H^{(\alpha)}}\right)\right)+\varphi_{*},
\end{aligned}
$$

where $\rho=\alpha \frac{p_{\varphi}}{2 H^{(\alpha)}}$. Here $H^{(\alpha)}, D, K$ and $p_{\varphi}$ are the integrals being generators of the $\mathfrak{s l}(2, \mathbb{R}) \oplus \mathfrak{u}(1)$ symmetry of the system (5.2), see eqs. (5.8), (5.9) below, and we see that the scattering angle is $\varphi_{\text {scat }}=\varphi(+\infty)-\varphi(-\infty)=\alpha \pi$ under assumption $p_{\varphi}>0$. Some pictures of the trajectory for different values of $\alpha$ are displayed in figure 1 .

From these figures one sees that when $\alpha$ is an even number, $\alpha=2 \ell$, the particle experiences a backward scattering, that corresponds to the scattering angle $2 \pi \ell$, while when $\alpha$ is odd, $\alpha=2 \ell+1$, the scattering angle is $2 \pi \ell+\pi$, and the particle continues asymptotic motion in the initial direction after realizing $\ell$ revolutions around the origin being the vertex of the cone. This was already observed in ref. [26]. ${ }^{3}$

To reveal the locally rectilinear character of geodesics in the case $\alpha>1$, it is convenient to consider the shape of trajectories in a cut and flattened cone represented by the angular sector $-\frac{\pi}{2} \alpha \leq \varphi \leq \frac{\pi}{2} \alpha$ (wedge) in which the symmetric points on the edges $\varphi=-\frac{\pi}{2} \alpha$ and $\varphi=\frac{\pi}{2} \alpha$ correspond to the cut line and must be identified. This is illustrated by figure 2 .

From the diagrams shown in figure 2 one reveals the following special features in the case of integer $\alpha$ :

1. If we look for the case in which the asymptotes of the trajectory are parallel to symmetry axis (going through the cone's vertex and the perihelion), we must impose the condition $\phi_{1}+\varepsilon=\pi / 2$ (where $\varepsilon=\phi_{n}$ ), which can be fulfilled if $\alpha=4 n$, with $n=1,2, \ldots$

2. When $\alpha=2(2 n+1)$, one has that the first incidence angle is given by $\pi /(2 n+1)$, which coincides with $\varphi_{s}$. This implies that the asymptotes of the path are parallel to the edges. This also includes the case $\alpha=2$ for which $n=0$ and the trajectory suffers no reflections at the edges.

3. When $\alpha=4 n-1$, the first incidence angle corresponds to $\frac{1}{4 n-1} \frac{\pi}{2}=\frac{\varphi_{s}}{4}$. When $\alpha=4 n+1$, the first incidence angle is given by $\frac{3}{4 n+1} \frac{\pi}{2}=\frac{3}{4} \varphi_{s}$.

\footnotetext{
${ }^{3}$ Note that our parameter $\alpha$ corresponds to $\alpha^{-1}$ in notations of [26], where only the case of positive mass density of the cosmic string (that corresponds to $\alpha>1$ values of our parameter) was considered.
} 


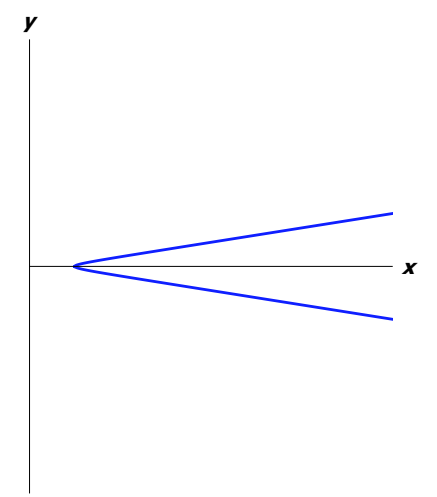

(a) $\alpha=1 / 10$

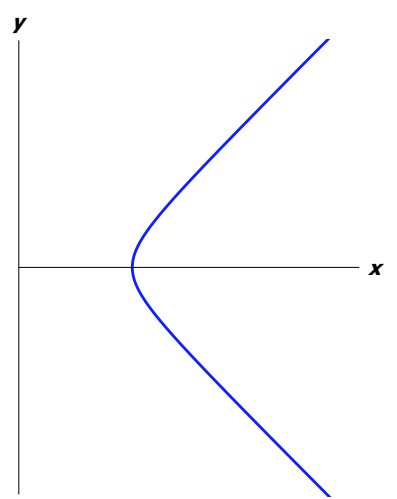

(b) $\alpha=1 / 2$

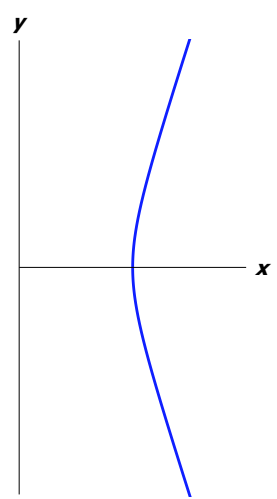

(c) $\alpha=4 / 5$

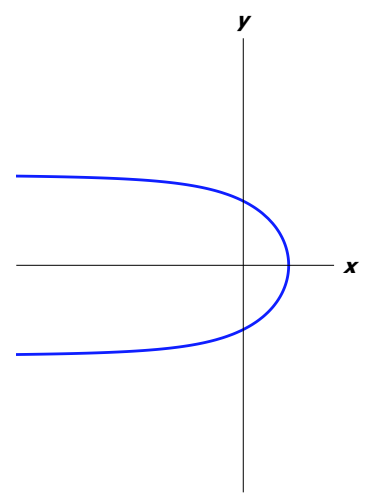

(d) $\alpha=2$

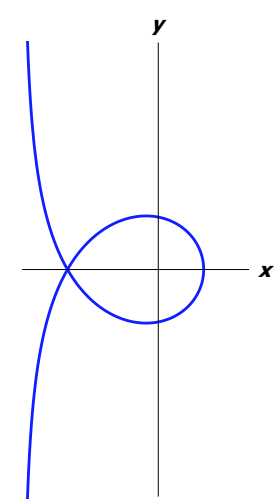

(e) $\alpha=3$

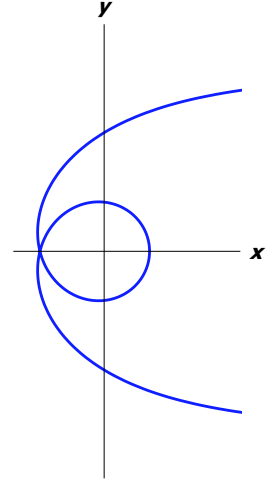

(f) $\alpha=4$

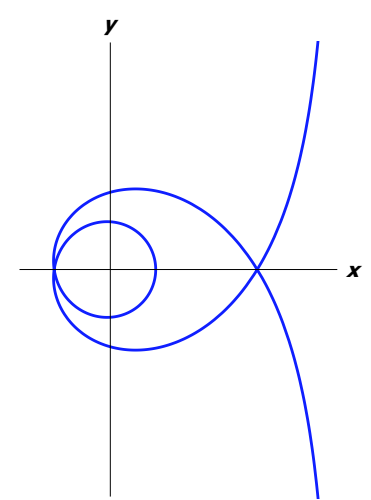

(g) $\alpha=5$

Figure 1. Some examples of the geodesics in the conical geometry. The axes are oriented so that the perihelion is on the positive side of the $x$-axis $\left(\varphi_{*}=0\right)$. From the first three figures one sees that for $0<\alpha<1$, the dynamics is somewhat similar to that in the Kepler-Coulomb problem in the case of repulsive potential. For the shown integer values of $\alpha$ the $t=-\infty$ and $t=+\infty$ straight line asymptotes coincide with a vertical line for odd values of $\alpha$, and are parallel horizontal lines for even values of $\alpha$.

Now, let's pass over to constructing the symmetry generators of the model by applying the transformation (5.3) to those of the Euclidean free particle analyzed in section 3. We start with the complex combinations of the locally defined translations and the Galilean boost generators,

$$
\begin{aligned}
& \Pi_{ \pm}=\Pi_{1} \pm i \Pi_{2}=\left(\frac{p_{r}}{\alpha} \pm i \frac{p_{\varphi}}{r}\right) e^{ \pm i \frac{\varphi}{\alpha}} \\
& \Xi_{ \pm}=\Xi_{1} \pm i \Xi_{2}=\left[\alpha m r-t\left(\frac{p_{r}}{\alpha} \pm i \frac{p_{\varphi}}{r}\right)\right] e^{ \pm i \frac{\varphi}{\alpha}} .
\end{aligned}
$$

Note that the presence of the factor $\alpha^{-1}$ in the integrals (5.6) and (5.7) implies that they are globally well-defined functions on the phase space only in the case of natural values of $\alpha^{-1}=k=1,2, \ldots$, and otherwise they are formal objects. In spite of this deficiency, these quantities will serve as the basis for constructing the globally well-defined in the phase 


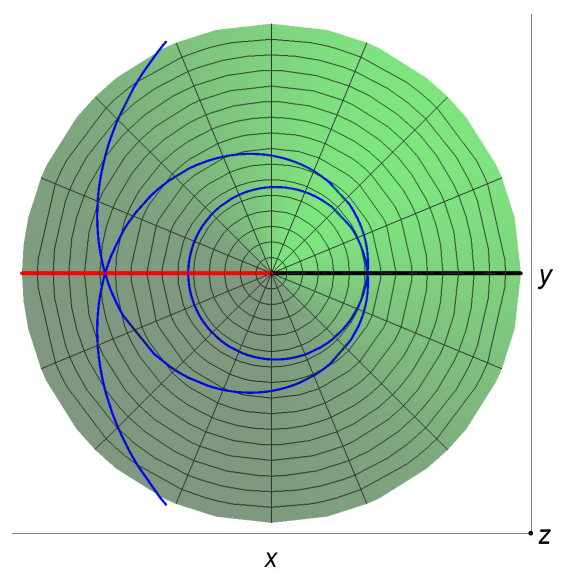

(a) Cutting the cone.

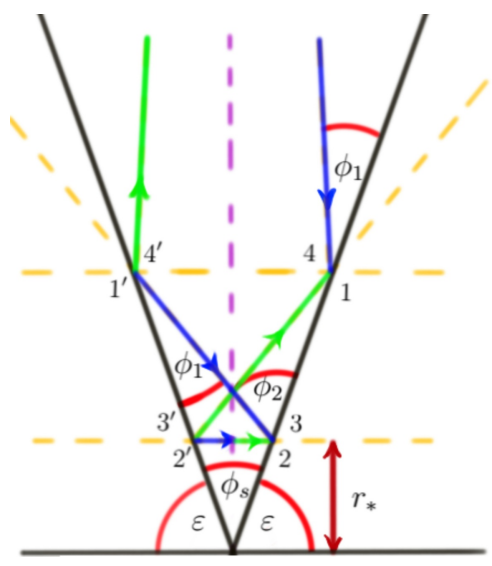

(b) Rectilinear geodesic on the cut and flattened cone.

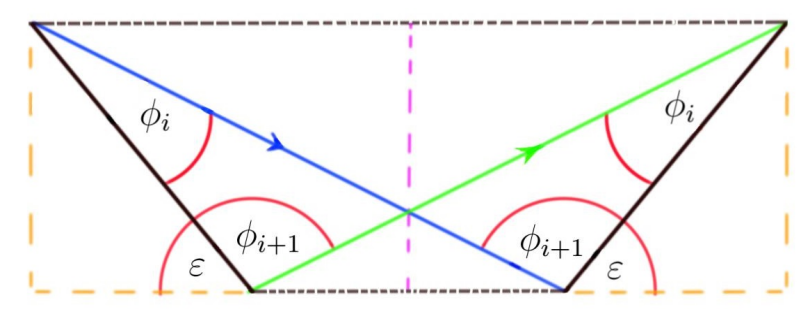

(c) Incidence and reflection angles at the edges of the angular sector.

Figure 2. Rectilinear geodesic motion in the cone. Figure 2a: to construct the flattened cone, we cut it along the straight line (shown in black), which is opposite (with respect to the cone symmetry axis) to the straight line (shown in red) going from the cone's vertex to the point of perihelion. As a result we obtain a flattened sector of the angle $\varphi_{s}=\frac{2 \pi}{\alpha}$, with identified edges. Figure 2b: the rectilinear character of geodesic in the obtained angular sector with identified edges. The incoming part of the trajectory (from infinity to the point of perihelion $r_{*}$ ) is represented in blue, while the outgoing part (from $r_{*}$ to infinity) is shown in green. When the particle arrives at the right edge in a point $i$ at an angle of incidence $\phi_{i}$, it emerges from the left edge in the point $i^{\prime}$ at the same angle, and continues its rectilinear path to the point $(i+1)$, and so on. In the general case the particle "hits" the edges several times. Note that by the construction, the lowest triangle on the diagram, appearing when $\alpha>2$, with the vertex corresponding to the cone's vertex and opposite side going through the perihelion $r_{*}$, is always isosceles. This implies that the last angle of incidence equals $\varepsilon=\pi\left(\frac{1}{2}-\frac{1}{\alpha}\right)$. Figure 2c: the shown geometric structure appears several times in accordance with the number of nonzero incidence angles. Using the geometric properties of the triangles, one has that $\phi_{i+1}=\phi_{i}-2 \varepsilon+\pi$, and making use of the fact that the last angle corresponds to $\varepsilon$, we obtain $\phi_{n-\ell}=\pi\left(\frac{1}{2}-\frac{2 \ell+1}{\alpha}\right)$ where $n$ represents the number of incidence angles (the number of "reflections" at the edges for $\alpha>2)$ and $\ell=0,1, \ldots, n-1$. From the condition $\phi_{1}>0$ we get that $2(2 n-1)<\alpha$. 
space symmetry generators of the system. For arbitrary values of $\alpha$ we have

$$
\begin{aligned}
H^{(\alpha)} & =\frac{1}{2 m} \Pi_{+} \Pi_{-}, & D & =\frac{1}{4 m}\left(\Xi_{+} \Pi_{-}+\Pi_{+} \Xi_{+}\right)=\frac{r p_{r}}{2}-H^{(\alpha)} t, \\
K & =\frac{1}{2 m} \Xi_{+} \Xi_{-}=\frac{m}{2} \alpha^{2} r^{2}-2 D t-H^{(\alpha)} t^{2}, & J_{0} & =\frac{i}{4 m}\left(\Xi_{+} \Pi_{-}-\Pi_{+} \Xi_{+}\right)=\frac{\alpha}{2} p_{\varphi},
\end{aligned}
$$

which are the well-defined angular-independent generators of the $\mathfrak{s l}(2, \mathbb{R}) \oplus \mathfrak{s o}(2)$ symmetry,

$$
\begin{aligned}
& \left\{D, H^{(\alpha)}\right\}=H^{(\alpha)}, \quad\{D, K\}=-K, \quad\left\{K, H^{(\alpha)}\right\}=2 D, \\
& \left\{J_{0}, H^{(\alpha)}\right\}=\left\{J_{0}, D\right\}=\left\{J_{0}, K\right\}=0,
\end{aligned}
$$

with the $\mathfrak{s l}(2, \mathbb{R})$ subalgebra Casimir element $D^{2}-H^{(\alpha)} K=-J_{0}^{2}$.

For rational values $\alpha=q / k, q, k=1,2, \ldots$, local integrals (5.6)-(5.7) can be used to construct the higher-order globally well-defined integrals

$$
\begin{aligned}
& \mathcal{O}_{\mu, \nu}^{ \pm}=\left(\Xi_{ \pm}\right)^{\mu}\left(\Pi_{ \pm}\right)^{\nu}, \quad \quad \mu=0,1, \ldots, q, \quad \nu=q-\mu, \\
& \mathcal{S}_{\mu^{\prime}, \nu^{\prime}}^{ \pm}=\left(\Xi_{ \pm}\right)^{\mu^{\prime}}\left(\Pi_{ \pm}\right)^{\nu^{\prime}}, \quad \quad \mu^{\prime}=0,1, \ldots, 2 q, \quad \nu^{\prime}=2 q-\mu^{\prime} \text {. }
\end{aligned}
$$

There are $2(q+1)$ conserved quantities of the type $\mathcal{O}_{\mu, \nu}^{ \pm}$and $2(2 q+1)$ conserved quantities of the type $\mathcal{S}_{\mu^{\prime}, \nu^{\prime}}^{ \pm}$, which have the angular dependence of the form $e^{ \pm i k \varphi}$ and $e^{ \pm i 2 k \varphi}$, respectively. So, these are globally well-defined functions in the phase space.

In the Euclidean case $q=k=1$, the integrals (5.12) correspond to the generators of the two-dimensional Heisenberg algebra (3.13), while the integrals (5.13) constitute the set of the second-order integrals $J_{0}, J_{ \pm}, T_{ \pm}, S_{ \pm}$defined by eqs. (3.2), (3.3). In the case of $q=1$ and $k=2,3, \ldots$, i.e. when $\alpha$ is a unit fraction $1 / k$ with $k>1$, the integrals $\Pi_{ \pm}$and $\Xi_{ \pm}$are well-defined functions in the corresponding phase space, and the set of integrals (5.12), (5.13) is similar to that of the free particle in Euclidean plane. Together with the conformal symmetry generators $H^{(\alpha)}, D$ and $K$, they generate the same Lie algebra as for the free particle in the plane since the canonical transformation (5.3) does not change the Poisson bracket relations.

To characterize the symmetry algebra for $q>1$, which will be nonlinear in the general case, it is necessary to calculate some Poisson bracket relations, and for this we use the identity $\left\{A, B^{n}\right\}=n\{A, B\} B^{n-1}$ together with the Poisson bracket relations

$$
\begin{aligned}
& \left\{\Xi_{ \pm}, \Pi_{\mp}\right\}=2 m \\
& \left\{H^{(\alpha)}, \Xi_{ \pm}\right\}=-\Pi_{ \pm}, \quad\left\{D, \Xi_{ \pm}\right\}=-\frac{1}{2} \Xi_{ \pm}, \quad\left\{J_{0}, \Xi_{ \pm}\right\}=\mp \frac{i}{2} \Xi_{ \pm}, \\
& \left\{K, \Pi_{ \pm}\right\}=\Xi_{ \pm}, \quad\left\{D, \Pi_{ \pm}\right\}=\frac{1}{2} \Pi_{ \pm}, \quad\left\{J_{0}, \Pi_{ \pm}\right\}=\mp \frac{i}{2} \Pi_{ \pm} .
\end{aligned}
$$

For integrals $\mathcal{O}_{\mu, \nu}^{ \pm}$we have then

$$
\begin{aligned}
& \left\{J_{0}, \mathcal{O}_{\mu, \nu}^{ \pm}\right\}=\mp i \frac{\mu+\nu}{2} \mathcal{O}_{\mu, \nu}^{ \pm}, \quad\left\{D, \mathcal{O}_{\mu, \nu}^{ \pm}\right\}=\frac{\nu-\mu}{2} \mathcal{O}_{\mu, \nu}^{ \pm}, \\
& \left\{H^{(\alpha)}, \mathcal{O}_{\mu, \nu}^{ \pm}\right\}=-\mu \mathcal{O}_{\mu-1, \nu+1}^{ \pm}, \quad\left\{K, \mathcal{O}_{\mu, \nu}^{ \pm}\right\}=\nu \mathcal{O}_{\mu+1, \nu-1}^{ \pm}, \quad\left\{\mathcal{O}_{\mu, \nu}^{ \pm}, \mathcal{O}_{\lambda, \sigma}^{ \pm}\right\}=0
\end{aligned}
$$


and by using the Jacobi identity we get

$$
\begin{aligned}
\left\{J_{0},\left\{\mathcal{O}_{\mu, \nu}^{+}, \mathcal{O}_{\lambda, \sigma}^{-}\right\}\right\} & =0, \quad\left\{D,\left\{\mathcal{O}_{\mu, \nu}^{+}, \mathcal{O}_{\lambda, \sigma}^{-}\right\}\right\}=\frac{\nu-\mu+\sigma-\lambda}{2}\left\{\mathcal{O}_{\mu, \nu}^{+}, \mathcal{O}_{\lambda, \rho}^{-}\right\} \\
\left\{H^{(\alpha)},\left\{\mathcal{O}_{\mu, \nu}^{+}, \mathcal{O}_{\lambda, \sigma}^{-}\right\}\right\} & \left.=-\lambda\left\{\mathcal{O}_{\mu, \nu}^{+}, \mathcal{O}_{\lambda-1, \sigma+1}^{-}\right\}-\mu\left\{\mathcal{O}_{\mu-1, \nu+1}^{+}, \mathcal{O}_{\lambda, \sigma}^{-}\right\}\right) \\
\left.\left\{K,\left\{\mathcal{O}_{\mu, \nu}^{+}, \mathcal{O}_{\lambda, \sigma}^{-}\right\}\right\}\right\} & =\sigma\left\{\mathcal{O}_{\mu, \nu}^{+}, \mathcal{O}_{\lambda+1, \sigma-1}^{-}\right\}+\nu\left\{\mathcal{O}_{\mu+1, \nu-1}^{+}, \mathcal{O}_{\lambda, \sigma}^{-}\right\}
\end{aligned}
$$

From relations (5.19) it follows that the Poisson brackets $\left\{\mathcal{O}_{\mu, \nu}^{+}, \mathcal{O}_{\lambda, \sigma}^{-}\right\}$must be functions of the globally well-defined generators $m, D, J_{0}, H^{(\alpha)}$ and $K$ since they are the only generators Poisson-commuting with $J_{0}$. We do not treat these functions as new, independent generators, but consider them as coefficients of a nonlinear algebra. On the other hand, all the generators $\mathcal{O}_{\mu, \nu}^{ \pm}$are eigenstates of the generators $D$ and $J_{0}$, in the sense of the Poisson bracket relations $\{I, A\}=\lambda A, I=D, J_{0}$. By taking their Poisson brackets with $H^{(\alpha)}$ and $K$, we generate the complete list of symmetry generators of this kind. Thus, the set $\mathcal{U}_{1}=\left\{H^{(\alpha)}, D, K, J_{0}, \mathcal{O}_{\mu, \nu}^{ \pm}\right\}$generates a finite nonlinear algebra.

In the same way, one can see that the integrals $\mathcal{S}_{\mu^{\prime}, \nu^{\prime}}^{ \pm}$satisfy the relations similar to those shown above for $\mathcal{O}_{\mu, \nu}^{ \pm}$but with the Greek indices changed for their primed versions. This implies that the set of generators $\mathcal{U}_{2}=\left\{H^{(\alpha)}, D, K, J_{0}, \mathcal{S}_{\mu^{\prime}, \nu^{\prime}}^{ \pm}\right\}$also produces a finite nonlinear algebra. To study what happens when we mix both sets of generators, we consider the relations

$$
\begin{aligned}
& \left\{D,\left\{\mathcal{S}_{\mu^{\prime}, \nu^{\prime}}^{ \pm}, \mathcal{O}_{\mu, \nu}^{\mp}\right\}\right\}=\frac{\mu-\nu-\mu^{\prime}-\nu^{\prime}}{2}\left\{\mathcal{S}_{\mu^{\prime}, \nu^{\prime}}^{ \pm}, \mathcal{O}_{\mu, \nu}^{\mp}\right\}, \\
& \left\{J_{0},\left\{\mathcal{S}_{\mu^{\prime}, \nu^{\prime}}^{ \pm}, \mathcal{O}_{\mu, \nu}^{\mp}\right\}\right\}=\mp i \frac{\mu+\nu}{2}\left\{\mathcal{S}_{\mu^{\prime}, \nu^{\prime}}^{ \pm}, \mathcal{O}_{\nu, \nu}^{\mp}\right\},
\end{aligned}
$$

where we have used the Jacobi identity one more time. Comparing the last relation with the first equation in (5.17), we deduce that in the general case, the integrals $\left\{\mathcal{S}_{\mu^{\prime}, \nu^{\prime}}^{ \pm}, \mathcal{O}_{\mu, \nu}^{\mp}\right\}$ must be functions of the integrals from the set $\mathcal{U}_{1}$. This means that the set of integrals $\mathcal{O}_{\mu, \nu}^{\mp}$ corresponds to generators of an ideal nonlinear subalgebra.

\subsection{Quantum case}

The quantum Hamiltonian of the system is constructed by using the Laplace-Beltrami operator,

$$
\hat{H}^{(\alpha)}=-\frac{\hbar^{2}}{2 m} \frac{1}{\sqrt{g}} \frac{\partial}{\partial x^{i}} \sqrt{g} g^{i j} \frac{\partial}{\partial x^{j}}=-\frac{\hbar^{2}}{2 m}\left(\frac{1}{\alpha^{2} r} \frac{\partial}{\partial r}\left(r \frac{\partial}{\partial r}\right)+\frac{1}{r^{2}} \frac{\partial^{2}}{\partial \varphi}\right) .
$$

The corresponding eigenstates and spectrum are given by

$$
\psi_{\kappa, l}^{ \pm}(r, \varphi)=\sqrt{\frac{\kappa}{2 \pi \alpha}} J_{\alpha l}(\kappa r) e^{ \pm i l \varphi}, \quad E=\frac{\hbar^{2} \kappa^{2}}{2 m \alpha^{2}}, \quad l=0,1, \ldots
$$

These eigenstates satisfy the orthogonality relation $\left\langle\psi_{\kappa, l}^{ \pm} \mid \psi_{\kappa^{\prime}, l^{\prime}}^{\mp}\right\rangle=\delta_{l l^{\prime}} \delta\left(\kappa-\kappa^{\prime}\right)$, with respect to the scalar product

$$
\left\langle\Psi_{1} \mid \Psi_{2}\right\rangle=\int_{V} \Psi_{1}^{*} \Psi_{2} \sqrt{g} d V=\int_{0}^{\infty} \alpha r d r \int_{0}^{2 \pi} d \varphi \Psi_{1}^{*} \Psi_{2}
$$


To address the problem of analyzing the quantum symmetry of the system, we consider the quantum versions of the formal integrals $\Pi_{ \pm}$and $\Xi_{ \pm}$, which have been the basis of the algebraic construction in the classical case. We start with

$$
\hat{\Pi}_{ \pm}=\hat{\Pi}_{1} \pm i \hat{\Pi}_{2}=e^{ \pm \frac{i \varphi}{2 \alpha}}\left(\frac{1}{\alpha} \hat{p}_{r} \pm \frac{i}{r} \hat{p}_{\varphi}\right) e^{ \pm \frac{i \varphi}{2 \alpha}}=-i \hbar \frac{1}{\alpha} e^{ \pm i \frac{\varphi}{\alpha}}\left(\frac{\partial}{\partial r} \pm i \frac{\alpha}{r} \frac{\partial}{\partial \varphi}\right)
$$

where $\hat{p}_{r}$ and $\hat{p}_{\varphi}$ are the radial and angular momentum operators introduced in (3.25). Operators $\hat{\Pi}_{ \pm}$are formal since their action on the eigenstates produces

$$
\begin{aligned}
& \hat{\Pi}_{ \pm} \psi_{\kappa, l}^{ \pm}(r, \varphi)=i \frac{\hbar \kappa}{\alpha} \sqrt{\frac{\kappa}{2 \pi \alpha}} J_{\alpha l+1}(\kappa r) e^{ \pm i\left(l+\frac{1}{\alpha}\right) \varphi}, \\
& \hat{\Pi}_{ \pm} \psi_{\kappa, l}^{\mp}(r, \varphi)=-i \frac{\hbar \kappa}{\alpha} \sqrt{\frac{\kappa}{2 \pi \alpha}} J_{\alpha l-1}(\kappa r) e^{ \pm i\left(l-\frac{1}{\alpha}\right) \varphi},
\end{aligned}
$$

from where we explicitly see that they cannot be physical operators for arbitrary values of $\alpha$ since in the general case they can produce the functions outside the Hilbert space generated by the states $\psi_{\kappa, l}^{ \pm}$.

We also consider the quantum versions of the Galilean boosts (5.7), corresponding to the operators

$$
\hat{\Xi}_{ \pm}=\alpha m r e^{ \pm i \frac{\varphi}{\alpha}} \pm t \hat{\Pi}_{ \pm}=e^{ \pm i \frac{\varphi}{\alpha}}\left(m \alpha r-i \hbar \frac{\kappa}{\alpha} t\left[\frac{\partial}{\partial(\kappa r)} \pm i \frac{\alpha}{\kappa r} \frac{\partial}{\partial \varphi}\right]\right) .
$$

Due to appearance of $\hat{\Pi}_{ \pm}$in (5.30), the operators $\hat{\Xi}_{ \pm}$inherit all the problems of the former operators.

Let us carefully study the case $\alpha=q / k$. As in the classical analysis of the previous subsection, we can consider the powers of the $\hat{\Pi}_{ \pm}$and $\hat{\Xi}_{ \pm}$operators. The action of $\left(\hat{\Pi}_{ \pm}\right)^{q}$ on the Hamiltonian eigenstates produces

$$
\begin{aligned}
& \left(\hat{\Pi}_{ \pm}\right)^{q} \psi_{\kappa, l}^{ \pm}(r, \varphi)=\left(i \frac{k \hbar \kappa}{q}\right)^{q} \sqrt{\frac{k \kappa}{2 \pi q}} J_{\frac{q}{k}(l+k)}(\kappa r) e^{ \pm i(l+k) \varphi}=\left(i \frac{k \hbar \kappa}{q}\right)^{q} \psi_{\kappa, l+k}^{ \pm}(r, \varphi) \\
& \left(\hat{\Pi}_{ \pm}\right)^{q} \psi_{\kappa, l}^{\mp}(r, \varphi)=\left(-i \frac{k \hbar \kappa}{q}\right)^{q} \sqrt{\frac{k \kappa}{2 \pi q}} J_{\frac{q}{k}(l-k)}(\kappa r) e^{ \pm i(l-k) \varphi} .
\end{aligned}
$$

Last equation means that the functions

$$
\left(\hat{\Pi}_{ \pm}\right)^{q} \psi_{\kappa, j}^{\mp}(r, \varphi) \propto J_{-\frac{q|k-j|}{k}}(\kappa r) e^{\mp i|k-j| \varphi}, \quad j=1, \ldots, k-1,
$$

are outside the Hilbert space since the index of the Bessel function is negative and noninteger. On the other hand, in the special case $k=1$ we have

$$
\begin{aligned}
& \left(\hat{\Pi}_{ \pm}\right)^{q} \psi_{\kappa, l}^{ \pm}(r, \varphi)=\left(i \frac{\hbar \kappa}{q}\right)^{q} \sqrt{\frac{\kappa}{2 \pi q}} J_{q(l+1)}(\kappa r) e^{ \pm i(l+1) \varphi}=\left(i \frac{\hbar \kappa}{q}\right)^{q} \psi_{\kappa, l+1}^{ \pm}(r, \varphi), \\
& \left(\hat{\Pi}_{ \pm}\right)^{q} \psi_{\kappa, l}^{\mp}(r, \varphi)=\left(-i \frac{\hbar \kappa}{q}\right)^{q} \sqrt{\frac{\kappa}{2 \pi q}} J_{q(l-1)}(\kappa r) e^{ \pm i(l-1) \varphi}=\left(-i \frac{\hbar \kappa}{q}\right)^{q} \psi_{\kappa, l-1}^{\mp}(r, \varphi) .
\end{aligned}
$$

Now, using the fact that $q l=\mathfrak{n}$ is a positive integer number and $J_{-\mathfrak{n}}(\zeta)=(-1)^{\mathfrak{n}} J_{\mathfrak{n}}(\zeta)$, one can show that $\psi_{\kappa, \mp l}^{ \pm}=(-1)^{l s} \psi_{\kappa, \pm l}^{\mp}$. This implies that the operators $\left(\hat{\Pi}_{ \pm}\right)^{q}$ always produce 
physical eigenstates in the case of integer values $\alpha=q$. They are a kind of the spectrum generating operators which allow us to change the quantum number $l$ of the states in \pm 1 without changing their energies.

As in the classical case, formal operators $\hat{\Pi}_{ \pm}$and $\hat{\Xi}_{ \pm}$are the basis in the construction of the symmetry algebra, and we can built the quantum version of the generators of the $\mathfrak{s o}(2,1) \oplus \mathfrak{u}(1)$ symmetry taking place for arbitrary values of $\alpha$. We have

$$
\begin{aligned}
\hat{H}^{(\alpha)} & =\frac{1}{2 m} \hat{\Pi}_{+} \hat{\Pi}_{-}, \quad \hat{D}=\frac{1}{4 m}\left(\hat{\Xi}_{+} \hat{\Pi}_{-}+\hat{\Pi}_{+} \hat{\Xi}_{-}\right)=\frac{\hbar}{2 i}\left(r \frac{\partial}{\partial r}+1\right)-\hat{H}^{(\alpha)} t, \\
\hat{K} & =\frac{1}{2 m} \hat{\Xi}_{-} \hat{\Xi}_{+}=\frac{m}{2} \alpha^{2} r^{2}-2 \hat{D} t-\hat{H}^{(\alpha)} t^{2}, \quad \hat{J}_{0}=\frac{1}{2}\left(\hat{\Xi}_{1} \hat{\Pi}_{2}-\hat{\Xi}_{2} \hat{\Pi}_{1}\right)=\frac{\alpha}{2} \hat{p}_{\varphi} .
\end{aligned}
$$

These operators satisfy the commutation relations

$$
\begin{aligned}
{\left[\hat{D}, \hat{H}^{(\alpha)}\right] } & =i \hbar \hat{H}^{(\alpha)}, \quad[\hat{D}, \hat{K}]=-i \hbar \hat{K}, \quad\left[\hat{K}, \hat{H}^{(\alpha)}\right]=2 i \hbar \hat{D} \\
{\left[\hat{J}_{0}, \hat{D}\right] } & =\left[\hat{J}_{0}, \hat{H}^{(\alpha)}\right]=\left[\hat{J}_{0}, \hat{K}\right]=0 .
\end{aligned}
$$

However, the analysis related to the operators $\hat{\Pi}_{ \pm}$shows us that in contrast with the classical case, only for $\alpha=q$ we can have a well-defined additional symmetry generators, that reveals a kind of the quantum anomaly in the case of rational non-integer values of $\alpha$. For $\alpha=q$, the corresponding symmetry operators are

$$
\begin{aligned}
\hat{\mathcal{O}}_{\mu, \nu}^{ \pm} & =\left(\hat{\Xi}_{ \pm}\right)^{\mu}\left(\hat{\Pi}_{ \pm}\right)^{\nu}, & \mu+\nu & =q . \\
\hat{\mathcal{S}}_{\mu^{\prime}, \nu^{\prime}}^{ \pm} & =\left(\hat{\Xi}_{ \pm}\right)^{\mu^{\prime}}\left(\hat{\Pi}_{ \pm}\right)^{\nu^{\prime}}, & \mu^{\prime}+\nu^{\prime} & =2 q,
\end{aligned}
$$

being the quantum counterpart of the classical integrals (5.12) and (5.13) with $k=1$. By means of the commutation relations

$$
\begin{aligned}
& {\left[\hat{\Xi}_{ \pm}, \hat{\Pi}_{\mp}\right]=2 i m \hbar, \quad\left[\hat{\Xi}_{ \pm}, \hat{\Pi}_{ \pm}\right]=0,} \\
& {\left[\hat{H}^{(\alpha)}, \hat{\Xi}_{ \pm}\right]=-i \hbar \hat{\Pi}_{ \pm}, \quad\left[\hat{D}, \hat{\Xi}_{ \pm}\right]=-\frac{i \hbar}{2} \hat{\Xi}_{ \pm}, \quad\left[\hat{J}_{0}, \hat{\Xi}_{ \pm}\right]= \pm \frac{\hbar}{2} \hat{\Xi}_{ \pm},} \\
& {\left[\hat{K}, \hat{\Pi}_{ \pm}\right]=i \hbar \hat{\Xi}_{ \pm}, \quad\left[\hat{D}, \hat{\Pi}_{ \pm}\right]=\frac{i \hbar}{2} \hat{\Pi}_{ \pm}, \quad\left[\hat{J}_{0}, \hat{\Pi}_{ \pm}\right]= \pm \frac{\hbar}{2} \hat{\Pi}_{ \pm}}
\end{aligned}
$$

and the commutator identity $\left[\hat{A}, \hat{B}^{n}\right]=\sum_{j=1}^{n} \hat{B}^{j-1}[\hat{A}, \hat{B}] \hat{B}^{n-j}$, it can be shown that the properties of the classical nonlinear algebra generated by the integrals $\mathcal{O}_{\mu, \nu}^{ \pm}$and $\mathcal{S}_{\mu^{\prime}, \nu^{\prime}}^{ \pm}$with $k=1$ are preserved at the quantum level.

Some important algebraic relations are

$$
\begin{aligned}
{\left[\hat{p}_{\varphi}, \hat{\mathcal{O}}_{\mu, \nu}^{ \pm}\right] } & = \pm \hbar \hat{\mathcal{S}}_{\mu, \nu}^{ \pm}, & {\left[\hat{p}_{\varphi}, \hat{\mathcal{S}}_{\mu^{\prime}, \nu^{\prime}}^{ \pm}\right] } & = \pm \hbar \hat{\mathcal{S}}_{\mu^{\prime}, \nu^{\prime}}^{ \pm} \\
{\left[\hat{D}, \hat{\mathcal{O}}_{\mu, \nu}^{ \pm}\right] } & =i \hbar \frac{\nu-\mu}{2} \hat{\mathcal{O}}_{\mu, \nu}^{ \pm}, & {\left[\hat{D}, \hat{\mathcal{S}}_{\mu, \nu}^{ \pm}\right] } & =i \hbar \frac{\nu^{\prime}-\mu^{\prime}}{2} \hat{\mathcal{S}}_{\mu^{\prime}, \nu^{\prime}}^{ \pm}
\end{aligned}
$$

From (5.44) we learn that the operators $\hat{\mathcal{O}}_{\mu, \nu}^{ \pm}\left(\hat{\mathcal{S}}_{\mu^{\prime}, \nu^{\prime}}^{ \pm}\right)$change the angular momentum quantum number by $\pm 1( \pm 2)$, and it its clear that operators $\hat{\mathcal{O}}_{0, q}^{ \pm}=\left(\hat{\Pi}_{ \pm}\right)^{q}$ are responsible for the infinite degeneracy of the spectrum of the system. 


\section{Harmonic oscillator in a cosmic string background}

To study the dynamics of the harmonic oscillator in a cosmic string background, we set the potential term in action (5.1) to be $V(\boldsymbol{r})=\frac{m \omega^{2}}{2} g_{i j} x_{i} x_{j}=\frac{1}{2} m \alpha^{2} \omega^{2} r^{2}$. We will see that the particle dynamics has special characteristics for different values of $\alpha$, and these peculiarities are coherently reflected in the classical orbits, in the spectra of the system at the quantum level, and in the construction of the symmetry algebra, which again will reveal the quantum anomaly phenomenon.

We also show that this system is related to the free motion in the conical geometry by means of the conformal bridge transformation, and we use this to reconstruct the properties of the complete classical symmetry algebra for the case $\alpha=q / k$ and the quantum version in the case $\alpha=q$.

\subsection{Classical case}

The Hamiltonian of the system corresponds to

$$
H_{\mathrm{os}}^{(\alpha)}=\frac{1}{2 m}\left(\frac{p_{r}^{2}}{\alpha^{2}}+\frac{p_{\varphi}^{2}}{r^{2}}\right)+\frac{m \alpha^{2} \omega^{2}}{2} r^{2}
$$

and as it happened with the free particle, it is directly related to the Hamiltonian of the harmonic oscillator in the Euclidean plane, which was reviewed in the subsection 3.2, by means of the local canonical transformation (5.3). From here, the solutions of the corresponding equations of motion are immediately obtained,

$$
\begin{aligned}
r^{2}(\varphi) & =\frac{p_{\varphi}^{2}}{m H_{\mathrm{os}}^{(\alpha)}}\left(1+\delta \cos \left(\frac{2}{\alpha}\left(\varphi-\varphi_{*}\right)\right)\right)^{-1}, \\
\varphi(\tau) & =\alpha \arctan \left(\frac{r_{+}}{r_{-}} \tan \left(\omega\left(\tau-\tau_{*}\right)\right)\right)+\varphi_{*}, \\
r^{2}(\tau) & =\frac{2}{m \omega^{2}}\left(\omega \mathcal{D} \sin (2 \omega \tau)+\omega^{2} \mathcal{K} \cos (2 \omega \tau)+H_{\mathrm{os}}^{(\alpha)} \sin ^{2}(\omega \tau)\right) .
\end{aligned}
$$

Here $\mathcal{D}$ and $\mathcal{K}$ are given by

$$
\begin{aligned}
& \mathcal{K}=\frac{m \alpha^{2}}{2} r^{2} \cos (2 \omega \tau)+\frac{1}{\omega^{2}} H_{\mathrm{os}}^{(\alpha)} \sin ^{2}(\omega \tau)-\frac{1}{2 \omega} r p_{r} \sin (2 \omega \tau), \\
& \mathcal{D}=\frac{1}{2} r p_{r} \cos (2 \omega \tau)-\frac{1}{2 \omega}\left(H_{\mathrm{os}}^{(\alpha)}-m \omega^{2} \alpha^{2} r^{2}\right) \sin (2 \omega \tau) .
\end{aligned}
$$

The quantities

$$
r_{ \pm}^{2}=\frac{H_{\mathrm{os}}^{(\alpha)}}{m \omega^{2} \alpha^{2}}(1 \pm \delta), \quad \delta=\sqrt{1-\left(\frac{\omega \alpha p_{\varphi}}{H_{\mathrm{os}}^{(\alpha)}}\right)^{2}}
$$

are identified with the radial turning points of the trajectory, and $\varphi_{*}$ and $\tau_{*}$ are the angular position of one of the radial minima of the trajectory and the corresponding moment of time when the particle is in that place.

From the corresponding solutions of the equation of motion one finds that the trajectory is closed if and only if $\alpha$ is a rational number. The images of the orbit for some rational and irrational values of $\alpha$ are shown in figure 3 . 


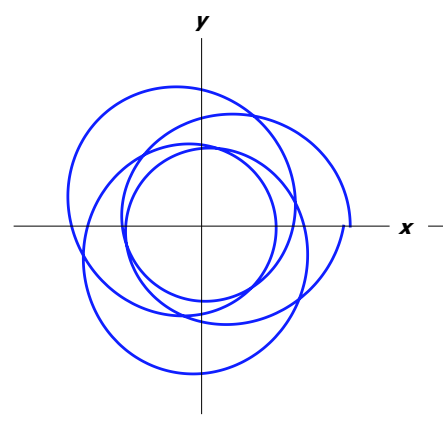

(a) $\alpha=e$

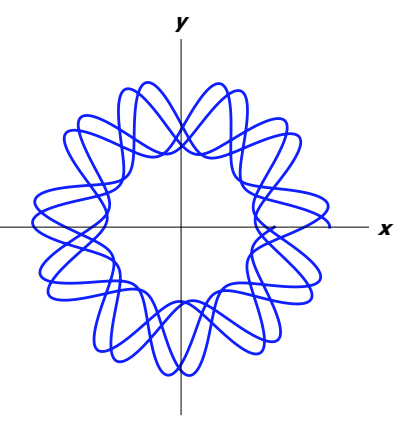

(b) $\alpha=1 / e$

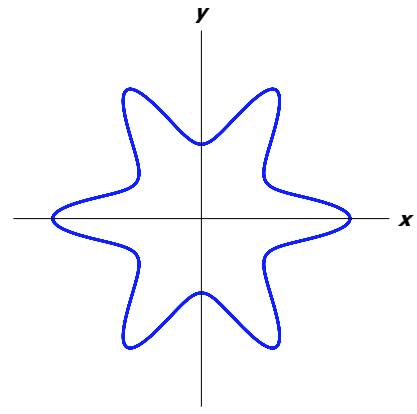

(c) $\alpha=1 / 3$

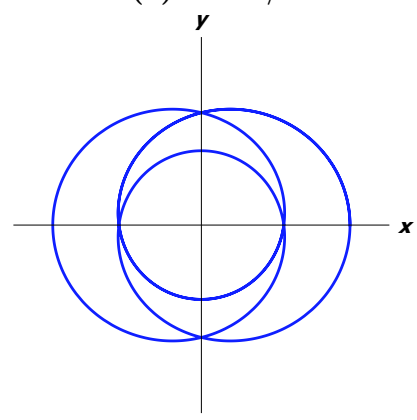

(f) $\alpha=3$

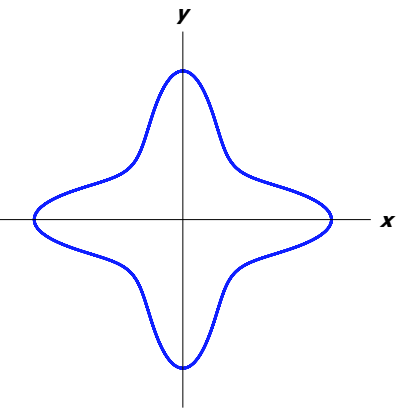

(d) $\alpha=1 / 2$

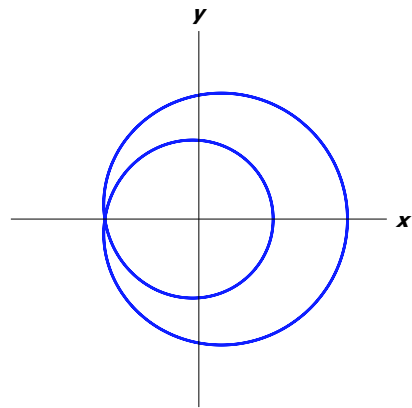

(g) $\alpha=4$

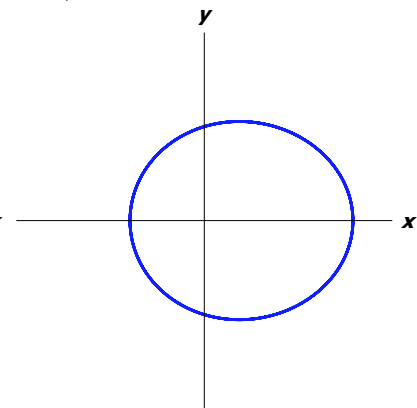

(e) $\alpha=2$

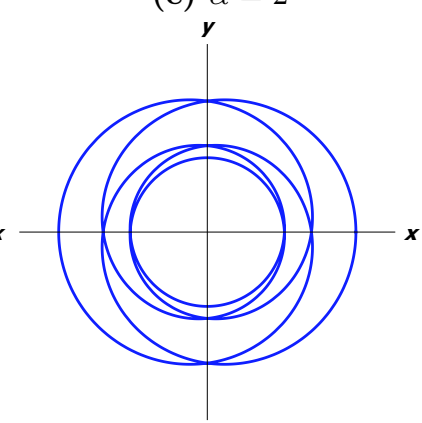

(h) $\alpha=5$

Figure 3. Images of the trajectory for some irrational and rational values of $\alpha$. From (6.2) one has that $r(\varphi)=r(\varphi+\alpha l \pi)$, implying that the number of radial minima $r_{-}$and maxima $r_{+}$depends on the value of $\alpha$. For $\alpha=1 / k$, one has $2 k$ minima and $2 k$ maxima. When $\alpha=2 n$, there is only one minimum and one maximum of $r$ on the orbit. When $\alpha=2 n+1$, there are two maxima and two minima, similarly to the case of the isotropic harmonic oscillator in the Euclidean plane. In general case, for $\alpha=q / k$ the number of maxima $/$ minima is $\mathscr{N}_{\max / \min }=k(q \bmod 2+1)$.

By using the canonical transformation (5.3), one can also obtain the dynamical integrals representing the classical analogues of the ladder operators in polar coordinates, which are:

$$
\begin{array}{ll}
\mathfrak{b}_{1}^{-}=\frac{1}{\sqrt{2}}\left(a_{1}^{-}-i a_{2}^{-}\right)=\frac{1}{2} e^{i\left(\omega t-\frac{\varphi}{\alpha}\right)}\left(\alpha \sqrt{m \omega} r+\frac{p_{\varphi}}{\sqrt{m \omega} r}+\frac{i p_{r}}{\alpha \sqrt{m \omega}}\right), & \mathfrak{b}_{1}^{+}=\left(\mathfrak{b}_{1}^{-}\right)^{*}, \\
\mathfrak{b}_{2}^{-}=\frac{1}{\sqrt{2}}\left(a_{1}^{-}+i a_{2}^{-}\right)=\frac{1}{2} e^{i\left(\omega t+\frac{\varphi}{\alpha}\right)}\left(\alpha \sqrt{m \omega} r-\frac{p_{\varphi}}{\sqrt{m \omega} r}+\frac{i p_{r}}{\alpha \sqrt{m \omega}}\right), & \mathfrak{b}_{2}^{+}=\left(\mathfrak{b}_{2}^{-}\right)^{*} .
\end{array}
$$


In resemblance with the free particle system in a conical geometry, we also note that these dynamical integrals are formal (locally defined only) functions in the respective phase space when $\alpha$ takes values different from $1 / k$. However, we can use these formal integrals to construct the well-defined generators of the $\mathfrak{s l}(2, \mathbb{R}) \oplus \mathfrak{u}(1)$ symmetry algebra of the system, which are given by

$$
\mathcal{J}_{0}=\frac{1}{2} \mathfrak{b}_{a}^{+} \mathfrak{b}_{a}^{-}=\frac{1}{2 \omega} H_{\mathrm{os}}^{(\alpha)}, \quad \mathcal{J}_{ \pm}=\mathfrak{b}_{1}^{ \pm} \mathfrak{b}_{2}^{ \pm}, \quad \mathcal{L}_{2}=\frac{1}{2}\left(\mathfrak{b}_{1}^{+} \mathfrak{b}_{1}^{-}-\mathfrak{b}_{2}^{+} \mathfrak{b}_{2}^{-}\right)=\frac{1}{2} \alpha p_{\varphi}
$$

In the context of the conformal bridge transformation, if we set the generators $H^{(\alpha)}$, $D$ and $K$ as those in (5.8)-(5.9), the transformation considered in section 4 produces the generators (6.10), in accordance with relations (4.11), (4.12) and the first equation in (4.15). To obtain the complete symmetry algebra for the case $\alpha=q / k$ we must apply the transformation to the higher-order generators (5.12) and (5.13). However, it is convenient first to consider the formal relations

$$
\begin{array}{rlrl}
\mathscr{T}(\tau, \beta, \delta, \gamma, t)\left(\Pi_{-}\right) & =-i \sqrt{2 m \omega} \mathfrak{b}_{1}^{-}, & & \mathscr{T}(\tau, \beta, \delta, \gamma, t)\left(\Pi_{+}\right)=-i \sqrt{2 m \omega} \mathfrak{b}_{2}^{-}, \\
\mathscr{T}(\tau, \beta, \delta, \gamma, t)\left(\Xi_{+}\right)=\sqrt{\frac{2 m}{\omega}} \mathfrak{b}_{1}^{+}, & \mathscr{T}(\tau, \beta, \delta, \gamma, t)\left(\Xi_{-}\right)=\sqrt{\frac{2 m}{\omega}} \mathfrak{b}_{2}^{+},
\end{array}
$$

where $\beta, \delta$ and $\gamma$ are still given by (4.10), and the formal equations (5.14)-(5.16) are employed. These equations facilitate the application of the conformal bridge transformation to the mentioned well-defined on the phase space integrals of motion. This yields us

$$
\begin{aligned}
& \mathscr{T}(\tau, \beta, \delta, \gamma, t)\left(\mathcal{O}_{\mu, \nu}^{ \pm}\right)=(-i)^{\nu}(m)^{\frac{q}{2}}(\omega)^{\frac{\mu-\nu}{2}} \mathcal{G}_{\mu, \nu}^{ \pm}, \\
& \mathscr{T}(\tau, \beta, \delta, \gamma, t)\left(\mathcal{S}_{\mu^{\prime}, \nu^{\prime}}^{ \pm}\right)=(-i)^{\nu}(m)^{\frac{q}{2}}(\omega)^{\frac{\mu-\nu}{2}} \mathcal{F}_{\mu^{\prime}, \nu^{\prime}}^{ \pm}
\end{aligned}
$$

where

$$
\mathcal{G}_{\mu, \nu}^{+}=\left(b_{1}^{+}\right)^{\mu}\left(b_{2}^{-}\right)^{\nu}, \quad\left(\mathcal{G}_{\mu, \nu}^{+}\right)^{*}=\mathcal{G}_{\nu, \mu}^{-} \quad \mathcal{F}_{\mu^{\prime}, \nu^{\prime}}^{+}=\left(b_{1}^{+}\right)^{\mu^{\prime}}\left(b_{2}^{-}\right)^{\nu^{\prime}}, \quad\left(\mathcal{F}_{\mu^{\prime}, \nu^{\prime}}^{+}\right)^{*}=\mathcal{F}_{\nu^{\prime}, \mu^{\prime}}^{-},
$$

and $\mu, \nu=0,1, \ldots q,\left(\mu^{\prime}, \nu^{\prime}=0,1, \ldots 2 q\right)$ satisfy the restriction $\mu+\nu=q\left(\mu^{\prime}+\nu^{\prime}=2 q\right)$. The properties of the symmetry algebra generated by these integrals of motion are retrieved directly from the symmetry algebra of the free particle moving in the same conical geometry. In summary we have:

- The set of generators $\mathcal{U}_{1}=\left\{\mathcal{J}_{0}, \mathcal{J}_{ \pm}, \mathcal{L}_{2}, \mathcal{G}_{\mu, \nu}^{ \pm}\right\}$produces an ideal nonlinear subalgebra. The (in general) dynamical integrals $\mathcal{G}_{\mu, \nu}^{ \pm}$are eigenstates of $i \mathcal{L}_{2}=i \alpha p_{\varphi} / 2$ with eigenvalue $\lambda= \pm q / 2$, in the sense of the Poisson bracket relation $\left\{\mathcal{L}_{2}, A\right\}=\lambda A$, and, therefore, they are eigenstates of $i p_{\varphi}$, with eigenvalue $\pm k$. In the same way, they are eigenstates of $H_{\mathrm{os}}^{(\alpha)}$ with eigenvalue $i \omega(\nu-\mu)$. Note that when $\nu=\mu=q / 2$ and $q$ is an even number, we have two true (not depending explicitly on time) integrals of motion for the system. Finally, the Poisson bracket action of generators $\mathcal{J}_{ \pm}$corresponds to

$$
\left\{\mathcal{J}_{-}, \mathcal{G}_{\mu, \nu}^{ \pm}\right\}=i \mu \mathcal{G}_{\mu-1, \nu+1}^{ \pm}, \quad\left\{\mathcal{J}_{+}, \mathcal{G}_{\mu, \nu}^{ \pm}\right\}=i \nu \mathcal{G}_{\mu+1, \nu-1}^{ \pm}
$$


- The integrals of the set $\mathcal{U}_{2}=\left\{\mathcal{J}_{0}, \mathcal{J}_{ \pm}, \mathcal{L}_{2}, \mathcal{F}_{\mu^{\prime}, \nu^{\prime}}^{ \pm}\right\}$also produce a nonlinear subalgebra, but which is not an ideal. In this case the (in general) dynamical integrals $\mathcal{F}_{\mu^{\prime}, \nu^{\prime}}^{ \pm}$ are eigenstates of $i \mathcal{L}_{2}$ with eigenvalue $\lambda= \pm q$, implying that with respect to $i p_{\varphi}$, the eigenvalues are $\pm 2 k$. These objects are also eigenstates of $H_{\mathrm{os}}^{(\alpha)}$, with eigenvalue $i \omega\left(\nu^{\prime}-\mu^{\prime}\right)$, and again, when $\nu^{\prime}=\mu^{\prime}=q$ we have two true integrals. In contrast with the previous case, these true integrals can be constructed for any natural value of $q$, but when $q$ is even, $q=2 \tilde{q}, \tilde{q}=1, \ldots$, we have the equalities $\mathcal{F}_{q, q}^{ \pm}=\left(\mathcal{G}_{\tilde{q}, \tilde{q}}^{ \pm}\right)^{2}$. The Poisson bracket action of the integrals $\mathcal{J}_{ \pm}$is

$$
\left\{\mathcal{J}_{-}, \mathcal{F}_{\mu^{\prime}, \nu^{\prime}}^{ \pm}\right\}=i \mu^{\prime} \mathcal{F}_{\mu^{\prime}-1, \nu^{\prime}+1}^{ \pm}, \quad\left\{\mathcal{J}_{+}, \mathcal{F}_{\mu^{\prime}, \nu^{\prime}}^{ \pm}\right\}=i \nu^{\prime} \mathcal{F}_{\mu^{\prime}+1, \nu^{\prime}-1}^{ \pm} .
$$

When $\alpha=1$, we obtain the harmonic oscillator in the Euclidean plane, and the dynamical integrals $\mathcal{G}_{\mu, \nu}^{ \pm}$correspond to the classical analogues of the ladder operators themselves. In this case, the true integrals $\mathcal{F}_{1,1}^{ \pm}$correspond to the $\mathcal{L}_{ \pm}$generators of the $\mathfrak{s u}(2)$ symmetry of the system, while $\mathcal{F}_{0,2}^{-}$and $\mathcal{F}_{2,0}^{+}$are the mutually complex conjugated dynamical integrals $\mathcal{B}^{ \pm}$, see section 3.2 . In the case $\alpha=1 / k$, with $k=2,3, \ldots$ we still have the same symmetry algebra as for the Euclidean case, since the generators $\mathfrak{b}_{a}^{ \pm}$are the globally well-defined functions in the phase space.

\subsection{Quantum case}

Here we study the quantum theory corresponding to the classical system discussed in the previous section. As we have seen, at the classical level the system has a large number of dynamical symmetries that are eigenstates of the Hamiltonian in the sense of the Poisson bracket relation $\left\{H_{\mathrm{os}}^{(\alpha)}, \mathcal{C}\right\}=\lambda \mathcal{C}$ when $\alpha$ is rational. Each of these integrals were obtained by applying the classical conformal bridge transformation to the classical free particle system symmetry generators in conical geometry, and in this section we follow the quantum version of that approach. In this context, and remembering that at the quantum level the system of the free particle in conical space reveals a quantum anomaly for rational, non-integer values of $\alpha$, it should not be surprising that this anomaly is also present in the harmonically trapped system, and in this section we show how this happens. As all the integrals that admit a well-defined quantum extensions must be eigenstates of the corresponding quantum Hamiltonian $\hat{H}_{\mathrm{os}}^{(\alpha)}$ in the sense of $\left[\hat{H}_{\mathrm{os}}^{(\alpha)}, \hat{\mathcal{C}}\right]=i \hbar \lambda \hat{\mathcal{C}}$, one concludes that the action of these operators at $\tau=0$ on a particular eigenstate of the system (and that actually is what we need to calculate according to eq. (3.28)) will produce another eigenstate. So, for the sake of simplicity, we assume that all the evolution parameters in dynamical integrals are zero from now on.

At the quantum level, the system is governed by the Hamiltonian operator

$$
\hat{H}_{\mathrm{os}}^{(\alpha)}=-\frac{\hbar^{2}}{2 m}\left(\frac{1}{\alpha^{2} r} \frac{\partial}{\partial r}\left(r \frac{\partial}{\partial r}\right)+\frac{1}{r^{2}} \frac{\partial^{2}}{\partial \varphi}\right)+\frac{\alpha^{2} m \omega^{2}}{2} r^{2},
$$

whose eigenstates and spectrum are given by

$$
\begin{aligned}
\psi_{n_{r}, l}^{ \pm}(r, \varphi) & =\left(\frac{m \omega \alpha^{2}}{\hbar}\right)^{\frac{1}{2}} \sqrt{\frac{n_{r} !}{2 \pi \alpha \Gamma\left(n_{r}+\alpha l+1\right)}} \zeta^{\alpha l} L_{n_{r}}^{(\alpha l)}\left(\zeta^{2}\right) e^{-\frac{\zeta^{2}}{2} \pm i l \varphi}, \zeta=\sqrt{\frac{m \alpha^{2} \omega}{\hbar} r}, \\
E_{n, l} & =\hbar \omega\left(2 n_{r}+\alpha l+1\right), \quad n_{r}, l=0,1, \ldots
\end{aligned}
$$


These eigenstates are orthonormal, $\left\langle\psi_{n_{r}, l}^{ \pm} \mid \psi_{n_{r}^{\prime}, l^{\prime}}^{\mp}\right\rangle=\delta_{n_{r}, n_{r}^{\prime}} \delta_{l, l^{\prime}}$, with respect to the scalar product (5.26).

Note that, contrary to the case of the free particle, the degeneracy of the energy levels depends on the value of $\alpha$. In the particular case of rational values $\alpha=q / k$ we distinguish two cases:

- When $q$ is even, $q=2 \tilde{q}$, the energy levels satisfy the relation

$$
E_{n_{r}+\tilde{q} j, l-k j}=E_{n_{r}, l}, \quad j=-\left[\frac{n_{r}}{\tilde{q}}\right], \ldots,\left[\frac{l}{k}\right],
$$

where [.] indicates the integer part of the quotient. This implies that all the eigenstates $\psi_{n_{r}+\tilde{q} j, l-k j}^{ \pm}$have the same energy eigenvalue. Counting the number of these eigenstates we get the following value for the degeneracy:

$$
g(N)=2\left[\frac{N}{\tilde{q}}\right]+1, \quad N=n_{r}+\frac{\tilde{q}}{k} l .
$$

- When $q$ is odd, we have

$$
E_{n_{r}+q j, l-2 k j}=E_{n_{r}, l}, \quad j=-\left[\frac{n_{r}}{q}\right], \ldots,\left[\frac{l}{2 k}\right],
$$

and the degeneracy is given by

$$
g(N)=\left[\frac{N}{q}\right]+1, \quad N=2 n_{r}+\frac{q}{k} l .
$$

Like the classical case, we can use the quantum version of the conformal bridge transformation to connect this system with the quantum version of the free particle in the conical geometry with the same value of $\alpha$. As we are interested in the operators at $\tau=0$, we consider the stationary conformal bridge transformation generated by $\hat{\mathfrak{S}}(0,0)=\hat{\mathfrak{S}}_{0}$, which produces

$$
\hat{\mathfrak{S}}_{0}\left(\hat{H}^{(\alpha)}\right) \hat{\mathfrak{S}}_{0}^{-1}=-\omega \hbar \hat{\mathcal{J}}_{-}, \quad \hat{\mathfrak{S}}_{0}\left(i \hat{D}_{0}\right) \hat{\mathfrak{S}}_{0}^{-1}=\hbar \hat{\mathcal{J}}_{0}, \quad \hat{\mathfrak{S}}_{0}\left(\hat{K}_{0}\right) \hat{\mathfrak{S}}_{0}^{-1}=\frac{\hbar}{\omega} \hat{\mathcal{J}}_{-},
$$

where

$$
\hat{\mathcal{J}}_{0}=\frac{1}{2 \hbar \omega} \hat{H}_{\mathrm{os}}^{(\alpha)}, \quad \hat{\mathcal{J}}_{ \pm}=-\frac{m \omega}{4 \hbar}\left(\hat{H}_{\mathrm{os}}^{(\alpha)}-m \omega^{2} \alpha^{2} r^{2} \pm \hbar \omega\left(r \frac{\partial}{\partial r}+1\right)\right) .
$$

Also, when the transformation acts on the formal operators $\hat{\Pi}_{ \pm}$and $\hat{\Xi}_{ \pm}$, one gets

$$
\begin{array}{ll}
\hat{\mathfrak{S}}_{0}\left(\hat{\Pi}_{-}\right) \hat{\mathfrak{S}}_{0}^{-1}=-i \sqrt{2 m \hbar \omega} \hat{\mathfrak{b}}_{1}^{-}, & \hat{\mathfrak{S}}_{0}\left(\hat{\Pi}_{+}\right) \hat{\mathfrak{S}}_{0}^{-1}=-i \sqrt{2 m \hbar \omega} \hat{\mathfrak{b}}_{2}^{-} \\
\hat{\mathfrak{S}}_{0}\left(\hat{\Xi}_{+}\right) \hat{\mathfrak{S}}_{0}^{-1}=\sqrt{\frac{2 m \hbar}{\omega}} \hat{\mathfrak{b}}_{1}^{+}, & \hat{\mathfrak{S}}_{0}\left(\hat{\Xi}_{-}\right) \hat{\mathfrak{S}}_{0}^{-1}=\sqrt{\frac{2 m \hbar}{\omega}} \hat{\mathfrak{b}}_{2}^{+},
\end{array}
$$

where

$$
\begin{array}{lll}
\hat{\mathfrak{b}}_{1}^{-}=\frac{1}{2} e^{-i \frac{\varphi}{\alpha}} \sqrt{\frac{m \omega}{\hbar}}\left(\alpha r+\frac{\hbar}{m \omega \alpha}\left(\frac{\partial}{\partial r}-\frac{i \alpha}{r} \frac{\partial}{\partial \varphi}\right)\right), & \hat{\mathfrak{b}}_{1}^{+}=\left(\hat{\mathfrak{b}}_{1}^{-}\right)^{\dagger}, \\
\hat{\mathfrak{b}}_{2}^{-}=\frac{1}{2} e^{i \frac{\varphi}{\alpha}} \sqrt{\frac{m \omega}{\hbar}}\left(\alpha r+\frac{\hbar}{m \omega \alpha}\left(\frac{\partial}{\partial r}+\frac{i \alpha}{r} \frac{\partial}{\partial \varphi}\right)\right), & \hat{\mathfrak{b}}_{2}^{+}=\left(\hat{\mathfrak{b}}_{2}^{-}\right)^{\dagger},
\end{array}
$$


are the formal dimensionless ladder operators in polar coordinates representation. In terms of them, the generators of the conformal algebra (6.26) and the quantum version of $\mathcal{L}_{2}$ take the form

$$
\begin{aligned}
\hat{\mathcal{J}}_{0} & =\frac{1}{4}\left(\left\{\hat{\mathfrak{b}}_{1}^{+}, \hat{\mathfrak{b}}_{1}^{-}\right\}+\left\{\hat{\mathfrak{b}}_{2}^{-}, \hat{\mathfrak{b}}_{2}^{+}\right\}\right), \quad \hat{\mathcal{J}}_{ \pm}=\hat{\mathfrak{b}}_{1}^{ \pm} \hat{\mathfrak{b}}_{2}^{ \pm}, \\
\hat{\mathcal{L}}_{2} & =\frac{1}{2}\left(\hat{\mathfrak{b}}_{1}^{+} \hat{\mathfrak{b}}_{1}^{-}-\hat{\mathfrak{b}}_{2}^{+} \hat{\mathfrak{b}}_{2}^{-}\right)=\frac{1}{2 \hbar} \hat{p}_{\varphi} .
\end{aligned}
$$

According to relation (4.22), the corresponding eigenstates of the form $\langle\boldsymbol{r} \mid \lambda\rangle$ we are looking for correspond to

$$
\Omega_{n_{r}, l}^{ \pm}(r, \varphi)=r^{2 n_{r}+\alpha l} e^{ \pm l \varphi}
$$

which satisfy the following set of equations,

$$
\begin{aligned}
2 i \hat{D}_{0} \Omega_{n_{r}, l}^{ \pm}(r, \varphi) & =\hbar\left(2 n_{r}+\alpha l+1\right) \Omega_{n_{r}, l}^{ \pm}(r, \varphi), \\
\hat{K}_{0} \Omega_{n_{r}, l}^{ \pm}(r, \varphi) & =\frac{m \alpha^{2}}{2} \Omega_{n_{r}+1, l}^{ \pm}(r, \varphi), \\
\hat{H}^{(\alpha)} \Omega_{n_{r}, l}^{ \pm}(r, \varphi) & =-\frac{2 \hbar^{2}}{m \alpha^{2}} n_{r}\left(n_{r}+\alpha l\right) \Omega_{n_{r}-1, l}^{ \pm}(r, \varphi), \\
\hat{\Xi}_{ \pm} \Omega_{n_{r}, l}^{ \pm}(r, \varphi) & =\alpha m \Omega_{n_{r}, l+\frac{1}{\alpha}}^{ \pm}(r, \varphi), \quad \hat{\Xi}_{ \pm} \Omega_{n_{r}, l}^{\mp}(r, \varphi)=\alpha m \Omega_{n_{r}+1, l-\frac{1}{\alpha}}^{\mp}(r, \varphi), \\
\hat{\Pi}_{ \pm} \Omega_{n_{r}, l}^{ \pm}(r, \varphi) & =-i \frac{2 \hbar n_{r}}{\alpha} \Omega_{n_{r}-1, l+\frac{1}{\alpha}}^{ \pm}(r, \varphi), \\
\hat{\Pi}_{ \pm} \Omega_{n_{r}, l}^{\mp}(r, \varphi) & =-i 2\left(n_{r}+\alpha l\right) \frac{\hbar}{\alpha} \Omega_{n_{r}, l-\frac{1}{\alpha}}^{\mp}(r, \varphi) .
\end{aligned}
$$

Here, the functions $\Omega_{0, l}^{ \pm}$are the zero energy eigenstates of $\hat{H}^{(\alpha)}$, but only the function $\Omega_{0,0}^{+}=\Omega_{0,0}^{-}=1$ is a physical eigenstate for the free particle system. Functions $\Omega_{n_{r}, l}^{ \pm}$are the rank $n_{r}$ Jordan states of zero energy which satisfy

$$
\begin{aligned}
\left(\hat{H}^{(\alpha)}\right)^{j} \Omega_{n_{r}, l}^{ \pm}(r, \varphi) & =\left(-2 \frac{\hbar}{m \alpha^{2}}\right)^{j}\left(n_{r}\right)_{j}\left(n_{r}+\alpha l\right)_{j} \Omega_{n_{r}-j, l}^{ \pm}(r, \varphi), \quad j=1, \ldots n_{r}, \\
(g)_{j} & =\prod_{i=0}^{j-1}(g-i) .
\end{aligned}
$$

Another remarkable property of these functions is that the eigenstates of the free particle admit the representation

$$
\psi_{\kappa, l}^{ \pm}(r, \varphi)=\sqrt{\frac{\kappa}{2 \pi \alpha}} \sum_{n_{r}=0}^{\infty} \frac{(-1)^{n_{r}}(\kappa / 2)^{2 n_{r}+\alpha l}}{n_{r} ! \Gamma\left(n_{r}+\alpha l+1\right)} \Omega_{n_{r}, l}^{ \pm}(r, \varphi) .
$$

By direct application of the stationary conformal bridge operator $\hat{\mathfrak{S}}_{0}$ to functions (6.33) one gets

$$
\begin{aligned}
\hat{\mathfrak{S}}_{0} \Omega_{n_{r}, l}^{ \pm}(r, \varphi) & =\mathcal{N}_{n_{r}, l} \psi_{n_{r}, l}^{ \pm}(r, \varphi), \\
\mathcal{N}_{n_{r}, l} & =(-1)^{n_{r}}\left(\frac{2 \hbar}{m \omega \alpha^{2}}\right)^{n_{r}+\frac{\alpha l}{2}} \sqrt{2 \alpha \pi n_{r} ! \Gamma\left(n_{r}+\alpha l+1\right)} .
\end{aligned}
$$


On the other hand, the action of the transformation on the free particle eigenstates produces the (non-normalized) coherent states of the system corresponding to

$$
\begin{aligned}
\Psi_{\kappa, \ell}^{ \pm}(r, \varphi) & =\hat{\mathfrak{S}}_{0} \psi_{\kappa, \ell}^{ \pm}(r, \varphi)=2^{\frac{1}{2}} e^{\mathcal{E}} \psi_{\kappa, \ell}^{ \pm}(\sqrt{2} r, \varphi) e^{-\frac{m \omega r^{2}}{2 \hbar}} \\
& =\sqrt{\kappa} \sum_{n_{r}=0}^{\infty} \frac{\mathcal{E}^{n_{r}+\frac{\alpha l}{2}}}{\sqrt{n_{r} ! \Gamma\left(n_{r}+\alpha l+1\right)}} \psi_{n_{r}, l}^{ \pm}(r, \varphi),
\end{aligned}
$$

where $\mathcal{E}=\frac{\hbar \kappa^{2}}{2 m \omega \alpha^{2}}$. These states satisfy the relation $\hat{\mathcal{J}}_{-} \Psi_{\kappa, \ell}^{ \pm}(r, \varphi)=-\mathcal{E} \Psi_{\kappa, \ell}^{ \pm}(r, \varphi)$.

From the equations (6.36) we obtain the action of generators $\hat{\mathcal{J}}_{ \pm}$,

$$
\begin{aligned}
& \hat{\mathcal{J}}_{+} \psi_{n_{r}, l}^{ \pm}(r, \varphi)=-\sqrt{\left(n_{r}+1\right)\left(n_{r}+\alpha l+1\right)} \psi_{n_{r}+1, l}^{ \pm}(r, \varphi), \\
& \hat{\mathcal{J}}_{-} \psi_{n_{r}, l}^{ \pm}(r, \varphi)=-\sqrt{n_{r}\left(n_{r}+\alpha l\right)} \psi_{n_{r}-1, l}^{ \pm}(r, \varphi),
\end{aligned}
$$

and from equations (6.37)-(6.39) we get the formal relations

$$
\begin{aligned}
& \hat{\mathfrak{b}}_{a}^{ \pm} \psi_{n_{r}, l}^{(a)}(r, \varphi)=\sqrt{\left(n_{r}+\alpha l+\beta_{ \pm}\right)} \psi_{n_{r}, l \pm \frac{l}{\alpha}}^{(a)}(r, \varphi), \\
& \hat{\mathfrak{b}}_{a}^{ \pm} \psi_{n_{r}, l}^{(b)}(r, \varphi)=-\sqrt{\left(n_{r}+\beta_{ \pm}\right)} \psi_{n_{r} \pm 1, l \mp \frac{l}{\alpha}}^{(b)}(r, \varphi),
\end{aligned}
$$

where $\psi_{n_{r}, l}^{(1)}=\psi_{n_{r}, l}^{+}, \psi_{n_{r}, l}^{(2)}=\psi_{n_{r}, l}^{-}$, and $\beta_{ \pm}=\frac{1}{2}(1 \pm 1)$. From these equations it becomes obvious that these operators cannot be physical for arbitrary values of $\alpha$, since they produce wave-functions outside the Hilbert space generated by the physical eigenstates $\psi_{n_{r}, l}^{ \pm}$. In fact, this is already observable from the equations (6.37)-(6.39), where it is seen that the produced functions on the right hand side do not satisfy some of the criteria imposed at the end of section 4: these functions are not single-valued in the angular coordinate, and some of them are singular at $r=0$.

Similarly to the free particle system in the conical space, we must study carefully the rational case $\alpha=q / k$, and to do that, it is enough to analyze the relations that imply a decrease in the angular momentum quantum number $l$ (the relations in which this number is increasing have no problems). First, consider the relation

$$
\left(\hat{\mathfrak{b}}_{a}^{-}\right)^{q} \psi_{n_{r}, l}^{(a)}(r, \varphi)=\sqrt{\frac{\Gamma\left(n_{r}+(q / k) l+1\right)}{\Gamma\left(n_{r}+(q / k) l-q+1\right)}} \psi_{n_{r}, l-k}^{(a)}(r, \varphi) .
$$

When $l<k$, that is $l-k=-j<0$, the explicit form of the function on the right hand side is

$$
\psi_{n_{r},-j}^{ \pm}(r, \varphi)=\left(\frac{m \omega q^{2}}{\hbar k^{2}}\right)^{\frac{1}{2}} \sqrt{\frac{k n_{r} !}{2 \pi q \Gamma\left(n_{r}-(q / k) j+1\right)}} \zeta^{-\frac{q}{k} j} L_{n_{r}}^{\left(-\frac{q}{k} j\right)}\left(\zeta^{2}\right) e^{-\frac{\zeta^{2}}{2} \mp i j \varphi},
$$

and because the upper index of the generalized Laguerre polynomial is negative and rational, we conclude that this function is outside the Hilbert space generated by the physical eigenstates, implying that the operators can not be observable. 
Now, in the particular case when $k=1$, the upper index in the generalized Laguerre polynomial is a negative integer number, and due to the identity

$$
\frac{(-\eta)^{i}}{i !} L_{n}^{(i-n)}(\eta)=\frac{(-\eta)^{n}}{n !} L_{i}^{(n-i)}(\eta), \quad i, n=0,1, \ldots,
$$

one gets that in the case $n_{r} \geq q l$,

$$
\psi_{n_{r},-l}^{ \pm}(r, \varphi)=(-1)^{q l} \psi_{n_{r}-q l, l}^{\mp}(r, \varphi),
$$

while for $n_{r}<q l$ the right hand side in eq. (6.51) vanishes due to the poles in the Gamma function. Therefore, eq. (6.50) has no problems when $k=1$. Note that in these cases the normalization factor (6.51) also equals zero by the same reason.

Now, we consider the relation

$$
\left(\hat{\mathfrak{b}}_{a}^{+}\right)^{q} \psi_{n_{r}, l}^{(b)}(r, \varphi)=(-1)^{q} \sqrt{\frac{\Gamma\left(n_{r}+1+q\right)}{\Gamma\left(n_{r}+1\right)}} \psi_{n_{r}+q, l-k}^{(b)}(r, \varphi),
$$

from where we see that the same problems for the cases $l<k$ appear again, and only in the case $k=1$ the relation (6.53) ensures that the operator always produces physical eigenstates.

The realized analysis of eqs. (6.50) and (6.54) reveals that, again, we face the problem of the quantum anomaly in the general case of rational values of $\alpha$, and we can construct a well-defined symmetry operators only in the case of integer values of $\alpha$, that we assume from now on.

Like the classical case, we construct the quantum symmetry generators by means of the conformal bridge transformation, obtaining

$$
\begin{aligned}
& \hat{\mathfrak{S}}_{0}\left(\hat{\mathcal{O}}_{\mu, \nu}^{ \pm}\right) \hat{\mathfrak{S}}_{0}^{-1}=(-i)^{\nu}(m \hbar)^{\frac{q}{2}}(\omega)^{\frac{\mu-\nu}{2}} \hat{\mathcal{G}}_{\mu, \nu}^{ \pm}, \\
& \hat{\mathfrak{S}}_{0}\left(\hat{\mathcal{S}}_{\mu^{\prime}, \nu^{\prime}}^{ \pm}\right) \hat{\mathfrak{S}}_{0}^{-1}=(-i)^{\nu}(m \hbar)^{q}(\omega)^{\frac{\mu^{\prime}-\nu^{\prime}}{2}} \hat{\mathcal{F}}_{\mu^{\prime}, \nu^{\prime}}^{ \pm}
\end{aligned}
$$

and the properties of the classical algebra (with $k=1$ ) are again preserved at the quantum level.

In conclusion of this discussion, let us make some comments with respect to the differences between the cases of even and odd values of $\alpha=q$. In the even case $q=2 \tilde{q}$, we have the true integrals of motion $\hat{\mathcal{G}}_{\tilde{q}, \tilde{q}}^{ \pm}$, whose explicit action on the eigenstates is

$$
\begin{aligned}
& \hat{\mathcal{G}}_{\tilde{q}, \tilde{q}}^{ \pm} \psi_{n_{r}, l}^{ \pm}(r, \varphi)=(-1)^{\tilde{q}} \sqrt{\frac{\Gamma\left(n_{r}+1\right) \Gamma\left(n_{r}+2 \tilde{q} l+1+\tilde{q}\right)}{\Gamma\left(n_{r}+1-\tilde{q}\right) \Gamma\left(n_{r}+2 \tilde{q} l+1\right)}} \psi_{n_{r}-\tilde{q}, l+1}^{ \pm}(r, \varphi), \\
& \hat{\mathcal{G}}_{\tilde{q}, \tilde{q}}^{\mp} \psi_{n_{r}, l}^{ \pm}(r, \varphi)=(-1)^{\tilde{q}} \sqrt{\frac{\Gamma\left(n_{r}+1+\tilde{q}\right) \Gamma\left(n_{r}+2 \tilde{q} l+1\right)}{\Gamma\left(n_{r}+1\right) \Gamma\left(n_{r}+2 \tilde{q} l+1-\tilde{q}\right)}} \psi_{n_{r}+\tilde{q}, l-1}^{ \pm}(r, \varphi) .
\end{aligned}
$$

From eq. (6.57) we learn that the operators $\hat{\mathcal{G}}_{\tilde{q}, \tilde{q}}^{ \pm}$annihilate the eigenstates $\psi_{n_{r}, l}^{ \pm}$with $n_{r}<$ $\tilde{q}-1$. Also, due to the relation (6.53), and the fact that $\psi_{n_{r}, 0}^{+}=\psi_{n_{r}, 0}^{-}:=\psi_{n_{r}, 0}$, both equations (6.57) and (6.58) are equivalent in the case $l=0$ and $n_{r}>\tilde{q}-1$. Otherwise, the pole in the Gamma function in (6.58) produces zero. One also notes that the index in the 
wave-functions that appears on the right hand side of these equations corresponds to the same index in the equations (6.21) for the cases $j= \pm 1$.

On the other hand, for the odd case $q=2 \tilde{q}+1$ we need to consider instead the integrals $\mathcal{F}_{q, q}^{ \pm}$which produce

$$
\begin{aligned}
& \hat{\mathcal{F}}_{q, q}^{ \pm} \psi_{n_{r}, l}^{ \pm}(r, \varphi)=(-1)^{q} \sqrt{\frac{\Gamma\left(n_{r}+1\right) \Gamma\left(n_{r}+q l+1+q\right)}{\Gamma\left(n_{r}+1-q\right) \Gamma\left(n_{r}+q l+1\right)}} \psi_{n_{r}-q, l+2}^{ \pm}(r, \varphi), \\
& \hat{\mathcal{F}}_{q, q}^{\mp} \psi_{n_{r}, l}^{ \pm}(r, \varphi)=(-1)^{q} \sqrt{\frac{\Gamma\left(n_{r}+1+q\right) \Gamma\left(n_{r}+q l+1\right)}{\Gamma\left(n_{r}+1\right) \Gamma\left(n_{r}+q l+1-q\right)}} \psi_{n_{r}+q, l-2}^{ \pm}(r, \varphi) .
\end{aligned}
$$

These equations reveal the properties similar to those described for the even case. The index in the resulting wave-function is in correspondence with the index in the equation (6.23).

Then, we conclude that the existence of the true (not depending explicitly on time) integrals $\hat{\mathcal{G}}_{\tilde{q}, \tilde{q}}^{ \pm}$and $\hat{\mathcal{F}}_{q, q}^{ \pm}$reflects the degeneracy of the system in the unique anomaly-free cases of the even and odd values of $\alpha$, respectively.

\section{Discussion and outlook}

The premise of our research here was that on the one hand, geometric properties of spacetime must be reflected in the intrinsic characteristics of the physical systems that inhabit it, and on the other hand, these peculiarities must be encoded in a set of well-defined integrals of motion. Bearing this in mind, we have studied different non-relativistic forms of dynamics (in the sense of Dirac [65]) associated with the $\mathfrak{s o}(2,1) \cong \mathfrak{s l}(2, \mathbb{R})$ conformal symmetry, namely, the free particle and the harmonic oscillator, on a cosmic string background [36-39]. This is the analogous problem of considering the non-relativistic conformal invariant dynamical models on a two-dimensional cone surface, the deficiency/excess angle of which is given in terms of the "geometrical parameter" $\alpha=1 /\left(1-\frac{4 \mu G}{c^{2}}\right)$, where $\mu$ is the linear mass density of the cosmic string [38, 45] which can be positive, or negative when topological defects in condensed matter physics and wormholes are considered [40-44]. Based on the previous observations related to the shape of the trajectories of the systems in this space $[26,28]$, one might assume that for some special values of the parameter $\alpha$, the systems may have well-defined hidden symmetry generators that adequately describe them. Our results confirm this hypothesis.

The main tools used in this investigation were a local canonical transformation and the conformal bridge transformation [55]. The first transformation relates the systems under investigation with their version in the flat Euclidean plane, while the second one allows us to map integrals from one form of conformal dynamics to another. The strategy was to start with a free particle in $\mathbb{R}^{2}(\alpha=1)$, from where we obtain, by a local canonical transformation, the solutions of the equations of motion and the formal integrals of the free system in the cone. These formal conserved quantities are the images of the Euclidean canonical momenta and the Galilean boosts, which are not globally well-defined functions in phase space for arbitrary $\alpha$ due to their peculiar angular dependence. However, no problems appear for $\alpha=1 / k, k=2,3, \ldots$, while in general case of rational values $\alpha=q / k$ 
with $q, k=1,2, \ldots$, these formal conserved quantities can be used to construct well-defined functions on phase space. After characterizing the complete symmetry algebra of the free particle in the cone, we have proceeded to apply the conformal bridge transformation in order to obtain the integrals and their symmetry algebra for the harmonic oscillator in the same geometric background.

In order to know what to expect for systems on the cone, it is instructive to make some comments about the models in $\mathbb{R}^{2}$. In the case of the free particle we have ten second-order integrals that generate the $\mathfrak{s p}(4, \mathbb{R})$ Lie algebra. Also, there are four firstorder integrals, namely the canonical momenta and the Galilean boosts, that produce the centrally extended two-dimensional Heisenberg algebra. The first order integrals generate an ideal sub-algebra of the complete Lie type symmetry of the system. In addition to the principal, conformal $\mathfrak{s o}(2,1)$ algebra, produced by the Hamiltonian $H$ and generators of dilatations, $D$, and special conformal transformations, $K$, one also can identify some other sub-algebraic structures:

- A secondary $\mathfrak{s l}(2, \mathbb{R})$ algebra, generated by the angular momentum and two dynamical integrals that commute with the dilatation generator.

- An $\mathfrak{s u}(2) \oplus \mathfrak{s u}(2)$ dynamical symmetry, produced by complex dynamical generators.

When considering the planar isotropic harmonic oscillator, one also finds 10 second-order symmetry generators. They produce an algebraic structure that is isomorphic to the $\mathfrak{s p}(4, \mathbb{R}$ ) algebra (up to complex linear combinations of generators). Furthermore, the system possesses an ideal two-dimensional Heisenberg sub-algebra, produced by the four first-order ladder operators. As for the free particle system, here we also have the principal $\mathfrak{s l}(2, \mathbb{R})$ algebra of the conformal Newton-Hooke symmetry generated by the Hamiltonian and the second order radial ladder operators. Other sub-algebraic structures which we would like to highlight are:

- The very well known $\mathfrak{s u}(2)$ symmetry, generated by the angular momentum and two other true integrals of motion that commute with the corresponding Hamiltonian.

- The $\mathfrak{s l}(2, \mathbb{R}) \oplus \mathfrak{s l}(2, \mathbb{R})$ symmetry, whose generators can be identified with the integrals of the associated Landau problem in symmetric gauge [55].

After applying the conformal bridge transformation to the Euclidean free particle system, we find that the principal $\mathfrak{s o}(2,1)$ conformal symmetry is mapped into the $\mathfrak{s l}(2, \mathbb{R})$ algebra of the conformal Newton-Hooke symmetry of the harmonic oscillator. In a similar way, the mentioned secondary $\mathfrak{s l}(2, \mathbb{R})$ symmetry of the free dynamics is mapped to the above-mentioned $\mathfrak{s u}(2)$ symmetry of the harmonically trapped particle; the complex algebra $\mathfrak{s u}(2) \oplus \mathfrak{s u}(2)$ of the free case is transformed into the $\mathfrak{s l}(2, \mathbb{R}) \oplus \mathfrak{s l}(2, \mathbb{R})$ sub-symmetry of the harmonically confined system, and there is a non-unitary correspondence between the Heisenberg algebras of both models. In the general case the conformal bridge transformation maps sub-algebras of one system into sub-algebras of the another. This happens due to its nature of the complex canonical transformation at the classical level, and of 
the non-unitary similarity transformation at the quantum level, which is the non-unitary automorphism of the conformal $\mathfrak{s o}(2,1) \cong \mathfrak{s l}(2, \mathbb{R})$ algebra [55].

It is important to reinforce the fact that in both cases the Lie algebraic structure described here is greater than the Schrödinger symmetry presented in Niederer's early works $[51,56]$. There, only the generators of the conformal symmetry, the Heisenberg symmetry and rotations were considered. Let us note here that at the quantum level, the ten second-order generators in both systems can be built by taking anti-commutators of the linear integrals. Then, if we consider a nonlocal operator of the rotation in $\pi$, $\mathscr{R}=\exp \left(i \pi \hat{p}_{\varphi}\right)$, due to relations $\mathscr{R} x_{i}=-x_{i} \mathscr{R}$ and $\mathscr{R}^{2}=1$, it can be identified as a grading operator. As a result, the Lie symmetry algebras we considered can be reinterpreted as Lie superalgebras $\mathfrak{o s p}(1 \mid 4, \mathbb{R})$ of the corresponding two-dimensional systems with no fermionic degrees of freedom [68]. This corresponds to the so-called systems with the bosonized (hidden) supersymmetry, see [69-74] and references therein. In one-dimensional quantum case, the origin of such type of hidden supersymmetries can be understood in terms of reduction of supersymmetric systems with fermionic degrees of freedom [72, 74]. It would be interesting to investigate in a similar way the origin of the indicated two-dimensional hidden (bosonized) supersymmetry.

The conformal and rotational symmetries of the free particle on the cone are described by the integrals which are well-defined phase space functions for arbitrary value of the geometric parameter $\alpha>0$, and generate the $\mathfrak{s o}(2,1) \oplus \mathfrak{u}(1)$ algebra. They are quadratic in the above-mentioned formal in general case basic integrals. For the case of rational $\alpha=q / k(q, k=1,2, \ldots$ with no common divisors), the formal basic generators can be used to build the new well-defined integrals of motion:

- When $q=1$ and $k=2,3, \ldots$ the symmetry algebra is the same as for the free particle in the flat Euclidean plane $(\alpha=1)$.

- When $q=2,3 \ldots$ and $k=1,2, \ldots$, it is possible to construct two different sets of higher-order integrals of motion: a set $\mathcal{U}_{1}$, consisting of $2 q+2$ integrals of order $q$, and the other set $\mathcal{U}_{2}$, which contains $4 q+2$ integrals of order $2 q$. We have verified that both sets generate independent finite nonlinear algebras, and together they produce a larger finite nonlinear algebraic structure. We have also shown that the nonlinear algebra generated by $\mathcal{U}_{1}$ is an ideal sub-algebra of the complete nonlinear symmetry.

After analyzing the classical system, we have considered the quantum case. The system can be quantized for arbitrary values of $\alpha$, and one could expect that for the rational case, the corresponding quantum versions of the (in general) higher-order hidden symmetry integrals will be the spectrum generating operators. However, we have revealed a quantum anomaly, since only in the case of integer values $\alpha=q=2,3, \ldots$ such spectrum generating integrals indeed can be constructed, while in the case of rational non-integer values of $\alpha$ the quantum analogs of the classically well-defined hidden symmetry generators take out the states from the physical Hilbert space.

Here, there is a couple of open interesting questions. First, knowing that via the corresponding non-relativistic limit $[57,58,61]$, one can relate conformal symmetry of the 
free particle on the cone with the corresponding Killing and conformal Killing vector fields of the cosmic string space-time background, a natural question is what geometrical objects correspond to the considered hidden symmetry generators. We speculate that they can be related to some Killing and conformal Killing tensors $[1,75]$ of the cosmic string space-time. Second, it would be interesting to look what happens with the quantum anomaly under perspective of unconventional boundary conditions, which were considered for quantum systems in the cone in [27].

Finally, we reconstruct the complete information on the symmetry algebra for the harmonic oscillator at the classical and quantum levels through the conformal bridge transformation. From here we have learned that the generators of the $\mathfrak{s l}(2, \mathbb{R}) \oplus \mathfrak{u}(1)$ algebra are well-defined for any value of $\alpha$, but only in the rational case $\alpha=q / k$ there is an extended set of the well-defined integrals of motion. This reflects the peculiarity of the geometry of the trajectories of the harmonically trapped particle on the cone: the trajectories are closed only when $\alpha=q / k$. The number of radial minima/maxima on the trajectory is given by $\mathscr{N}_{\min / \max }=k(q \bmod 2+1)$. In particular, for $k=1$ one has $\mathscr{N}_{\min / \max }=1$ when $\alpha$ is even and $\mathscr{N}_{\min / \max }=2$ when $\alpha$ is odd. At the quantum level it is seen that the value of $\alpha$ explicitly determines the degeneracy of the energy spectrum of the system, while the symmetry operators and their action on the corresponding eigenstates of the system are obtained directly from those of the free particle by employing the quantum version of the conformal bridge transformation. From there, the quantum anomaly is revealed automatically: only when $\alpha=q=2,3, \ldots$, it is possible to have the well-defined higher-order differential operators corresponding to hidden symmetries, which reflect the spectral degeneracy.

In conclusion, let us indicate some other problems for which the results and ideas employed in this article can be used.

First, we note that the local canonical transformation that relates the cone with the flat Euclidean plane can be applied for the analysis of other central potentials on the conical background. One can expect that the well-defined hidden symmetry generators in such systems can appear only at special values of $\alpha$, and that quantum anomaly can also emerge there. In particular, all the analysis presented here can immediately be transferred and generalized for the case of conformal mechanics on the cone as it was done in [55] in the flat Euclidean plane. In the same vein, the results of [55] can be employed and generalized immediately for the Landau problem on the cone.

Second, it would be interesting to employ the conformal bridge transformation for the systems in different geometries, such as the Lobachevsky plane and the non-commutative plane. These both geometries are used in the description of anyons [76-78], and on the other hand, one has to bare in mind that anyons can directly be related with the cone geometry [79-81].

Finally, as it was shown in [55], the non-unitary generator $\hat{\mathfrak{S}}$ of the conformal bridge transformation is the fourth order root of the space reflection operator $\mathcal{P}$, which in the present two-dimensional case has to be substituted for the above-mentioned non-local operator $\mathscr{R}=\exp \left(i \pi \hat{p}_{\varphi}\right)$. At the same time, it is easy to see from the explicit form (4.20) of $\hat{\mathfrak{S}}$ in terms of the $\mathfrak{s l}(2, \mathbb{R})$ generators of conformal symmetry that it is $\mathcal{P} \mathcal{T}$ symmetric, where $\mathcal{T}$ corresponds to the time reversal (anti-linear) transformation [82-86]. Taking also into 
account that its classical analog generates complex canonical transformations in the phase space, and that conformal symmetry plays important role in some $\mathcal{P} \mathcal{T}$-symmetric systems with peculiar properties [86-88], it would be very interesting to look at the conformal bridge transformations in the light of the $\mathcal{P} \mathcal{T}$ symmetry.

\section{Acknowledgments}

The work was partially supported by the FONDECYT Project 1190842 and the DICYT Project 042131P_POSTDOC.

Open Access. This article is distributed under the terms of the Creative Commons Attribution License (CC-BY 4.0), which permits any use, distribution and reproduction in any medium, provided the original author(s) and source are credited.

\section{References}

[1] M. Cariglia, Hidden Symmetries of Dynamics in Classical and Quantum Physics, Rev. Mod. Phys. 86 (2014) 1283 [arXiv:1411.1262] [INSPIRE].

[2] J. de Boer, F. Harmsze and T. Tjin, Nonlinear finite $W$ symmetries and applications in elementary systems, Phys. Rept. 272 (1996) 139 [hep-th/9503161] [INSPIRE].

[3] J. Beckers, Y. Brihaye and N. Debergh, On realizations of nonlinear Lie algebras by differential operators and some physical applications, J. Phys. A 32 (1999) 2791 [hep-th/9803253] [INSPIRE].

[4] W. Pauli, Über das Wasserstoffspektrum vom Standpunkt der neuen Quantenmechanik, Z. Phys. 36 (1926) 336 [inSPIRE].

[5] D.M. Fradkin, Three-dimensional isotropic harmonic oscillator and $\mathrm{SU}_{3}$, Am. J. Phys. 33 (1965) 207.

[6] M.S. Plyushchay and A. Wipf, Particle in a self-dual dyon background: hidden free nature, and exotic superconformal symmetry, Phys. Rev. D 89 (2014) 045017 [arXiv:1311.2195] [INSPIRE].

[7] L. Inzunza, M.S. Plyushchay and A. Wipf, Hidden symmetry and (super)conformal mechanics in a monopole background, JHEP 04 (2020) 028 [arXiv:2002.04341] [INSPIRE].

[8] S. Wojciechowski, Superintegrability of the Calogero-Moser system, Phys. Lett. A 95 (1983) 279 .

[9] V.B. Kuznetsov, Hidden symmetry of the quantum Calogero-Moser system, Phys. Lett. A 218 (1996) 212 [solv-int/9509001] [INSPIRE].

[10] S.W. Hawking, Black hole explosions, Nature 248 (1974) 30 [INSPIRE].

[11] W.G. Unruh, Notes on black hole evaporation, Phys. Rev. D 14 (1976) 870 [InSPIRE].

[12] R. Britto-Pacumio, J. Michelson, A. Strominger and A. Volovich, Lectures on Superconformal Quantum Mechanics and Multi-Black Hole Moduli Spaces, NATO Sci. Ser. C 556 (2000) 255 [hep-th/9911066] [INSPIRE].

[13] P. Claus, M. Derix, R. Kallosh, J. Kumar, P.K. Townsend and A. Van Proeyen, Black holes and superconformal mechanics, Phys. Rev. Lett. 81 (1998) 4553 [hep-th/9804177] [INSPIRE]. 
[14] J.A. de Azcarraga, J.M. Izquierdo, J.C. Perez Bueno and P.K. Townsend, Superconformal mechanics and nonlinear realizations, Phys. Rev. D 59 (1999) 084015 [hep-th/9810230] [INSPIRE].

[15] G.W. Gibbons and P.K. Townsend, Black holes and Calogero models, Phys. Lett. B 454 (1999) 187 [hep-th/9812034] [INSPIRE].

[16] V. de Alfaro, S. Fubini and G. Furlan, Conformal Invariance in Quantum Mechanics, Nuovo Cim. A 34 (1976) 569 [INSPIRE].

[17] A. Galajinsky, O. Lechtenfeld and K. Polovnikov, $\mathcal{N}=4$ superconformal Calogero models, JHEP 11 (2007) 008 [arXiv:0708.1075] [INSPIRE].

[18] N. Kozyrev, S. Krivonos, O. Lechtenfeld and A. Sutulin, SU(2|1) supersymmetric mechanics on curved spaces, JHEP 05 (2018) 175 [arXiv: 1712.09898] [INSPIRE].

[19] B. Carter, Axisymmetric Black Hole Has Only Two Degrees of Freedom, Phys. Rev. Lett. 26 (1971) 331 [INSPIRE].

[20] G.W. Gibbons, R.H. Rietdijk and J.W. van Holten, SUSY in the sky, Nucl. Phys. B 404 (1993) 42 [hep-th/9303112] [INSPIRE].

[21] M. Cariglia, Quantum mechanics of Yano tensors: Dirac equation in curved spacetime, Class. Quant. Grav. 21 (2004) 1051 [hep-th/0305153] [INSPIRE].

[22] V.P. Frolov and D. Kubiznak, Hidden Symmetries of Higher Dimensional Rotating Black Holes, Phys. Rev. Lett. 98 (2007) 011101 [gr-qc/0605058] [INSPIRE].

[23] V.P. Frolov and D. Kubiznak, Higher-Dimensional Black Holes: Hidden Symmetries and Separation of Variables, Class. Quant. Grav. 25 (2008) 154005 [arXiv:0802.0322] [INSPIRE].

[24] V. Frolov, P. Krtous and D. Kubiznak, Black holes, hidden symmetries, and complete integrability, Living Rev. Rel. 20 (2017) 6 [arXiv:1705.05482] [INSPIRE].

[25] G. 't Hooft, Nonperturbative Two Particle Scattering Amplitudes in $(2+1)$-Dimensional Quantum Gravity, Commun. Math. Phys. 117 (1988) 685 [INSPIRE].

[26] S. Deser and R. Jackiw, Classical and Quantum Scattering on a Cone, Commun. Math. Phys. 118 (1988) 495 [INSPIRE].

[27] B.S. Kay and U.M. Studer, Boundary conditions for quantum mechanics on cones and fields around cosmic strings, Commun. Math. Phys. 139 (1991) 103 [InSPIRE].

[28] C. Furtado and F. Moraes, Harmonic oscillator interacting with conical singularities, J. Phys. A 33 (2000) 5513 [inSPIRE].

[29] J.L.A. Coelho and R.L.P.G. Amaral, Coulomb and quantum oscillator problems in conical spaces with arbitrary dimensions, J. Phys. A 35 (2002) 5255 [gr-qc/0111114] [INSPIRE].

[30] C.C. Barros Jr., Quantum mechanics in curved space-time, Eur. Phys. J. C 42 (2005) 119 [physics/0409064] [INSPIRE].

[31] G. De A. Marques, V.B. Bezerra and S.G. Fernandes, Exact solution of the Dirac equation for a Coulomb and scalar potentials in the gravitational field of a cosmic string, Phys. Lett. A 341 (2005) 39 [INSPIRE].

[32] K. Kowalski and J. Rembieliński, On the dynamics of a particle on a cone, Annals of Physics 329 (2013) 146 [arXiv: 1304.4412] [INSPIRE]. 
[33] F.M. Andrade and E.O. Silva, Effects of spin on the dynamics of the 2D Dirac oscillator in the magnetic cosmic string background, Eur. Phys. J. C 74 (2014) 3187 [arXiv:1403.4113] [INSPIRE].

[34] M. Hosseinpour, F.M. Andrade, E.O. Silva and H. Hassanabadi, Scattering and bound states for the Hulthén potential in a cosmic string background, Eur. Phys. J. C 77 (2017) 270 [Erratum ibid. 77 (2017) 373] [arXiv: 1608.03558] [INSPIRE].

[35] F. Ahmed, Relativistic quantum dynamics of spin-0 massive charged particle in the presence of external fields in $4 D$ curved space-time with a cosmic string, Eur. Phys. J. Plus 135 (2020) 108 [arXiv: 1910.12700] [INSPIRE].

[36] T.W.B. Kibble, Topology of Cosmic Domains and Strings, J. Phys. A 9 (1976) 1387 [INSPIRE].

[37] A. Vilenkin, Gravitational Field of Vacuum Domain Walls and Strings, Phys. Rev. D 23 (1981) 852 [INSPIRE].

[38] A. Vilenkin, Cosmic Strings and Domain Walls, Phys. Rept. 121 (1985) 263 [INSPIRE].

[39] J.S. Dowker, Quantum Field Theory on a Cone, J. Phys. A 10 (1977) 115 [inSPIRE].

[40] M. Visser, Traversable wormholes: Some simple examples, Phys. Rev. D 39 (1989) 3182 [arXiv:0809.0907] [INSPIRE].

[41] J.G. Cramer, R.L. Forward, M.S. Morris, M. Visser, G. Benford and G.A. Landis, Natural wormholes as gravitational lenses, Phys. Rev. D 51 (1995) 3117 [astro-ph/9409051] [INSPIRE].

[42] M.O. Katanaev and I.V. Volovich, Theory of defects in solids and three-dimensional gravity, Annals Phys. 216 (1992) 1 [INSPIRE].

[43] G.E. Volovik, The universe in a helium droplet Oxford Science Publications (2003) [DOI].

[44] N.S. Manton, Five Vortex Equations, J. Phys. A 50 (2017) 125403 [arXiv:1612.06710] [INSPIRE].

[45] D.D. Sokolov and A.A. Starobinsky, On the structure of curvature tensor on conical singularities, Dokl. Akad. Nauk 234 (1977) 1043 [Sov. Phys. Dokl. 22 (1977) 312].

[46] M. Aryal, L.H. Ford and A. Vilenkin, Cosmic Strings and Black Holes, Phys. Rev. D 34 (1986) 2263 [INSPIRE].

[47] C. Furtado, B.G.C. da Cunha, F. Moraes, E.R. Bezerra de Mello and V.B. Bezerra, Landau levels in the presence of disclinations, Phys. Lett. A 195 (1994) 90 [InSPIRE].

[48] S.N. Solodukhin, The Conical singularity and quantum corrections to entropy of black hole, Phys. Rev. D 51 (1995) 609 [hep-th/9407001] [INSPIRE].

[49] M.G. Germano, V.B. Bezerra and E.R. Bezerra de Mello, Gravitational effects due to a cosmic string in Schwarzschild space-time, Class. Quant. Grav. 13 (1996) 2663 [INSPIRE].

[50] E.R.B. de Mello and A.A. Saharian, Vacuum polarization induced by a cosmic string in anti-de Sitter spacetime, J. Phys. A 45 (2012) 115002 [arXiv:1110.2129] [INSPIRE].

[51] U. Niederer, The maximal kinematical invariance group of the harmonic oscillator, Helv. Phys. Acta 46 (1973) 191 [InSPIRE].

[52] P.D. Alvarez, J. Gomis, K. Kamimura and M.S. Plyushchay, $(2+1) D$ Exotic Newton-Hooke Symmetry, Duality and Projective Phase, Annals Phys. 322 (2007) 1556 [hep-th/0702014] [INSPIRE]. 
[53] A. Galajinsky, Conformal mechanics in Newton-Hooke spacetime, Nucl. Phys. B 832 (2010) 586 [arXiv: 1002.2290] [INSPIRE].

[54] K. Andrzejewski, Conformal Newton-Hooke algebras, Niederer's transformation and Pais-Uhlenbeck oscillator, Phys. Lett. B 738 (2014) 405 [arXiv:1409.3926] [InSPIRE].

[55] L. Inzunza, M.S. Plyushchay and A. Wipf, Conformal bridge between asymptotic freedom and confinement, Phys. Rev. D 101 (2020) 105019 [arXiv:1912.11752] [INSPIRE].

[56] U. Niederer, The maximal kinematical invariance group of the free Schrödinger equation., Helv. Phys. Acta 45 (1972) 802 [inSPIRE].

[57] C.R. Hagen, Scale and conformal transformations in galilean-covariant field theory, Phys. Rev. D 5 (1972) 377 [INSPIRE].

[58] A.O. Barut, Conformal group $\rightarrow$ Schrödinger group $\rightarrow$ dynamical group - the maximal kinematical group of the massive Schrödinger particle, Helv. Phys. Acta 46 (1973) 496.

[59] C. Leiva and M.S. Plyushchay, Conformal symmetry of relativistic and nonrelativistic systems and AdS/CFT correspondence, Annals Phys. 307 (2003) 372 [hep-th/0301244] [INSPIRE].

[60] M. Henkel and J. Unterberger, Schrödinger invariance and space-time symmetries, Nucl. Phys. B 660 (2003) 407 [hep-th/0302187] [INSPIRE].

[61] D.T. Son, Toward an AdS/cold atoms correspondence: A Geometric realization of the Schrödinger symmetry, Phys. Rev. D 78 (2008) 046003 [arXiv:0804.3972] [INSPIRE].

[62] A. Bagchi and R. Gopakumar, Galilean Conformal Algebras and AdS/CFT, JHEP 07 (2009) 037 [arXiv: 0902 .1385] [INSPIRE].

[63] M.S. Plyushchay, Quantization of the classical $\mathrm{SL}(2, \mathbb{R})$ system and representations of $\mathrm{SL}(2, \mathbb{R})$ group, J. Math. Phys. 34 (1993) 3954 [INSPIRE].

[64] V. Bargmann, Irreducible unitary representations of the Lorentz group, Annals Math. 48 (1947) 568 [INSPIRE].

[65] P.A.M. Dirac, Forms of Relativistic Dynamics, Rev. Mod. Phys. 21 (1949) 392 [inSPIRE].

[66] F. Correa, V. Jakubsky and M.S. Plyushchay, PT-symmetric invisible defects and confluent Darboux-Crum transformations, Phys. Rev. A 92 (2015) 023839 [arXiv:1506.00991] [INSPIRE].

[67] L. Inzunza and M.S. Plyushchay, Klein four-group and Darboux duality in conformal mechanics, Phys. Rev. D 99 (2019) 125016 [arXiv:1902.00538] [INSPIRE].

[68] M. de Crombrugghe and V. Rittenberg, Supersymmetric Quantum Mechanics, Annals Phys. 151 (1983) 99 [INSPIRE].

[69] M.S. Plyushchay, Deformed Heisenberg algebra, fractional spin fields and supersymmetry without fermions, Annals Phys. 245 (1996) 339 [hep-th/9601116] [INSPIRE].

[70] M. Plyushchay, Hidden nonlinear supersymmetries in pure parabosonic systems, Int. J. Mod. Phys. A 15 (2000) 3679 [hep-th/9903130] [InSPIRE].

[71] F. Correa and M.S. Plyushchay, Hidden supersymmetry in quantum bosonic systems, Annals Phys. 322 (2007) 2493 [hep-th/0605104] [INSPIRE].

[72] V. Jakubsky, L.-M. Nieto and M.S. Plyushchay, The origin of the hidden supersymmetry, Phys. Lett. B 692 (2010) 51 [arXiv:1004.5489] [INSPIRE].

[73] R. Bonezzi, O. Corradini, E. Latini and A. Waldron, Quantum Mechanics and Hidden Superconformal Symmetry, Phys. Rev. D 96 (2017) 126005 [arXiv:1709.10135] [inSPIRE]. 
[74] L. Inzunza and M.S. Plyushchay, Hidden superconformal symmetry: Where does it come from?, Phys. Rev. D 97 (2018) 045002 [arXiv:1711.00616] [INSPIRE].

[75] M. Crampin, Hidden symmetries and killing tensors, Rept. Math. Phys. 20 (1984) 31.

[76] M.S. Plyushchay, The Model of relativistic particle with torsion, Nucl. Phys. B 362 (1991) 54 [INSPIRE].

[77] P.A. Horvathy and M.S. Plyushchay, Non-relativistic anyons, exotic Galilean symmetry and noncommutative plane, JHEP 06 (2002) 033 [hep-th/0201228] [INSPIRE].

[78] P.A. Horvathy and M.S. Plyushchay, Anyon wave equations and the noncommutative plane, Phys. Lett. B 595 (2004) 547 [hep-th/0404137] [INSPIRE].

[79] J.M. Leinaas and J. Myrheim, On the theory of identical particles, Nuovo Cim. B 37 (1977) 1.

[80] J.M. Leinaas and J. Myrheim, Intermediate statistics for vortices in superfluid films, Phys. Rev. B 37 (1988) 9286 [INSPIRE].

[81] R. MacKenzie and F. Wilczek, Peculiar Spin and Statistics in Two Space Dimensions, Int. J. Mod. Phys. A 3 (1988) 2827 [InSPIRE].

[82] C.M. Bender and S. Boettcher, Real spectra in nonHermitian Hamiltonians having PT symmetry, Phys. Rev. Lett. 80 (1998) 5243 [physics/9712001] [INSPIRE].

[83] A. Mostafazadeh, PseudoHermiticity versus PT symmetry. The necessary condition for the reality of the spectrum, J. Math. Phys. 43 (2002) 205 [math-ph/0107001] [INSPIRE].

[84] C.M. Bender, Making sense of non-Hermitian Hamiltonians, Rept. Prog. Phys. 70 (2007) 947 [hep-th/0703096] [INSPIRE].

[85] A. Fring, PT-symmetric deformations of integrable models, Phil. Trans. Roy. Soc. Lond. A 371 (2013) 20120046 [arXiv: 1204.2291] [INSPIRE].

[86] P. Dorey, C. Dunning and R. Tateo, From PT-symmetric quantum mechanics to conformal field theory, Pramana 73 (2009) 217 [arXiv:0906.1130] [InSPIRE].

[87] J. Mateos Guilarte and M.S. Plyushchay, Perfectly invisible $\mathcal{P} \mathcal{T}$-symmetric zero-gap systems, conformal field theoretical kinks, and exotic nonlinear supersymmetry, JHEP 12 (2017) 061 [arXiv: 1710.00356] [INSPIRE].

[88] J. Mateos Guilarte and M.S. Plyushchay, Nonlinear symmetries of perfectly invisible PT-regularized conformal and superconformal mechanics systems, JHEP 01 (2019) 194 [arXiv: 1806.08740] [INSPIRE]. 